\title{
Progressive Structural Defects in Canine Centronuclear Myopathy Indicate a Role for HACD1 in Maintaining Skeletal Muscle Membrane Systems
}

Gemma L. Walmsley, ${ }^{*}$ Stéphane Blot, ${ }^{\dagger \dagger}$ Kerrie Venner, ${ }^{\S}$ Caroline Sewry, ${ }^{\top}$ Jocelyn Laporte,,$\|$ Jordan Blondelle, $^{\dagger}$ Inès Barthélémy, ${ }^{\dagger \ddagger}$ Marie Maurer, ${ }^{\dagger \ddagger}$ Nicolas Blanchard-Gutton, ${ }^{\dagger \ddagger}$ Fanny Pilot-Storck, ${ }^{\dagger \ddagger}$ Laurent Tiret, ${ }^{\dagger \ddagger}$ and Richard J. Piercy*

\begin{abstract}
From the Comparative Neuromuscular Diseases Laboratory, * Department of Clinical Sciences and Services, Royal Veterinary College, London, United Kingdom; French National Institute of Health and Medical Research (Inserm), ${ }^{\dagger}$ Mondor Institute of Biomedical Research (IMRB), U955-E10 Biology of the Neuromuscular System, Créteil, France; the University of Paris East, ${ }^{\ddagger}$ Alfort School of Veterinary Medicine (EnvA), Maisons-Alfort, France; the Electron Microscopy Unit, ${ }^{\S}$ Institute of Neurology, London, United Kingdom; the Dubowitz Neuromuscular Centre, ${ }^{\uparrow}$ University College London Institute of Child Health and Great Ormond Street Hospital, London, United Kingdom; and the Department of Translational Medicine and Neurogenetics, Institute of Genetics and Cellular and Molecular Biology (IGBMC), Inserm U964, CNRS UMR7104, Strasbourg University, Illkirch, France
\end{abstract}

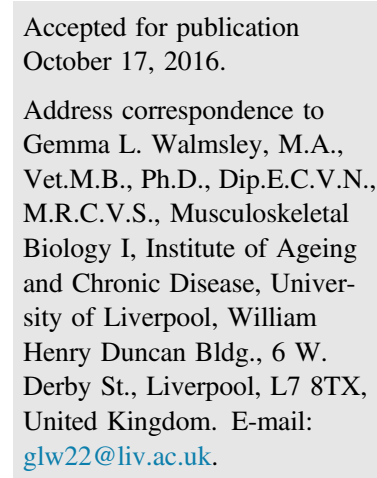

\begin{abstract}
Mutations in HACD1/PTPLA cause recessive congenital myopathies in humans and dogs. HydroxyacylCoA dehydratases are required for elongation of very long chain fatty acids, and HACD1 has a role in early myogenesis, but the functions of this striated muscle-specific enzyme in more differentiated skeletal muscle remain unknown. Canine HACD1 deficiency is histopathologically classified as a centronuclear myopathy (CNM). We investigated the hypothesis that muscle from HACD1-deficient dogs has membrane abnormalities in common with CNMs with different genetic causes. We found progressive changes in tubuloreticular and sarcolemmal membranes and mislocalized triads and mitochondria in skeletal muscle from animals deficient in HACD1. Furthermore, comparable membranous abnormalities in cultured HACD1-deficient myotubes provide additional evidence that these defects are a primary consequence of altered HACD1 expression. Our novel findings, including T-tubule dilatation and disorganization, associated with defects in this additional CNM-associated gene provide a definitive pathophysiologic link with these disorders, confirm that dogs deficient in HACD1 are relevant models, and strengthen the evidence for a unifying pathogenesis in CNMs via defective membrane trafficking and excitation-contraction coupling in muscle. These results build on previous work by determining further functional roles of HACD1 in muscle and provide new insight into the pathology and pathogenetic mechanisms of HACD1 CNM. Consequently, alterations in membrane properties associated with $H A C D 1$ mutations should be investigated in humans with related phenotypes. (Am J Pathol 2017, 187: 441-456; http://dx.doi.org/10.1016/j.ajpath.2016.10.002)
\end{abstract}

Centronuclear myopathies (CNMs) are a genetically heterogeneous group of congenital myopathies characterized clinically by paresis and skeletal muscle atrophy and histologically by abnormal centralization of myonuclei. ${ }^{1,2}$ Genes classically associated with human CNMs produce proteins involved in membrane trafficking or excitation-contraction coupling. ${ }^{3}$ Principally, these proteins include mutations in myotubularin (MTM1), a phosphoinositide phosphatase ${ }^{4}$; amphiphysin 2 (BIN1), which promotes and senses membrane curvature ${ }^{5}$;
Supported by The Myotubular Trust project grant BB/F016891/1 (R.J.P.), Biotechnology and Biological Sciences Research Council studentship grant BB/F016891/1 (G.L.W., R.J.P.), French National Research Agency grant ANR-12-JSV1-0005 (L.T., S.B.), French Association Against Myopathies grants 14577, 15882, 16143 and 17124 (L.T., S.B.), and CNM Project and the Alliance program grant 22866ZM/10.020 (L.T., S.B.).

Disclosures: None declared.

Current address of G.L.W., Musculoskeletal Biology I, Institute of Ageing and Chronic Disease, University of Liverpool, Liverpool, United Kingdom. 
dynamin 2 (DNM2), a protein involved in membrane tubulation and fission ${ }^{6}$; and the skeletal muscle ryanodine receptor (RYR1), the sarcoplasmic reticulum (SR) calcium release channel. ${ }^{7}$ Other human CNMs are associated with mutations in $C C D C 78,{ }^{8}$ SPEG,${ }^{9}$ and $T T N .{ }^{10}$

T-tubules are complex skeletal muscle membrane systems that conduct action potentials deep within the myofiber and near the internal sarcoplasmic stores at the calcium release unit or triad. Several causative genes for CNM produce proteins that associate with T-tubules; hence, the severe weakness that occurs in CNMs might result from altered morphologic features of T-tubule or SR membranes, altered coupling between the voltage-gated dihyropyridine receptor (DHPR) and ryanodine receptor (RyR1) calcium release channel, or more direct effects on RyR1 function or expression. ${ }^{11-22}$ Additional membrane defects at the sarcolemma, myofiber hypotrophy, and mislocalization of other proteins involved in membrane repair and recycling all strengthen the case for a more generalized derangement of membrane trafficking pathways in CNMs. ${ }^{14,18,19,21,23}$

Canine $H A C D 1$ CNM, an autosomal recessive condition that was recognized in Labrador retrievers 40 years ago, is the most common inherited myopathy in the breed. ${ }^{24-26}$ A colony of affected dogs was established in France in 1992, enabling more detailed evaluation, and the condition is now widely accepted as a CNM based on numerous clinical and pathologic features in common with CNMs in humans. ${ }^{25,27-29}$ Affected dogs do not develop myotatic reflexes and have markedly reduced muscle mass, hypotonia, and paresis compared with littermates from 1 month of age. Clinical signs are initially progressive but can stabilize at approximately 1 year of age. ${ }^{27-30}$ Their muscle exhibits several characteristic and progressive features: an early and marked heterogeneity in fiber size with altered oxidative staining followed by type 1 myofiber predominance and centralization of nuclei and fibrosis. ${ }^{27,28,31}$ Linkage analysis implicated a short interspersed nuclear element insertion in the PTPLA gene (recently renamed $H A C D 1$ ) as the causative mutation that alters splicing and reduces expression of normal transcripts. ${ }^{29}$ These transcripts include a full-length, muscle-specific isoform named HACD1-fl (hereafter, for simplicity, termed HACD1) and a shorter, ubiquitous isoform named HACD1-d5. ${ }^{29}$

Recently, HACD1 enzyme deficiency was also implicated in a novel human autosomal recessive, congenital myopathy in an extended, consanguineous Bedouin family. ${ }^{32}$ HACDl is highly expressed in developing and mature striated muscles $^{33-35}$ and encodes a 3-hydroxyacyl-CoA dehydratase enzyme with a role in very-long-chain fatty acid (VLCFA) biosynthesis, as part of the endoplasmic reticulum-bound, elongase complex. ${ }^{34,36,37}$ VLCFAs are metabolized in muscle as energy sources but, more importantly, are constituents of various membrane lipids, including phospholipids and sphingolipids, which have essential roles in membrane structure, fluidity, intracellular signaling, and membrane trafficking. ${ }^{38,39}$ In vitro, HACD1-deficient myoblasts have impaired myogenesis ${ }^{35,40}$ and, more specifically, defective myoblast fusion, likely because of altered sarcolemmal lipid content. ${ }^{40}$ In yeast and plants, HACD1 homologues (Phs1 and PASTICCINO2, respectively) are essential for growth and development. ${ }^{37,41,42}$

We investigated the hypothesis that canine $H A C D 1 \mathrm{CNM}$ recapitulates prominent features previously found in related human myopathies and have documented common findings of tubuloreticular disorganization and more general membrane abnormalities combined with mislocalization of other CNM proteins. ${ }^{11-22}$ Our work strengthens the evidence for a common pathogenesis between this myopathy and human CNMs, validates HACDI CNM in dogs as a CNM model, and reveals a previously unidentified role for $H A C D 1$ in muscle. Documenting pathologic consequences associated with HACD1 deficiency in CNM provides further insight into CNM pathogenesis and confirms a general regulatory role for HACD1 in membrane composition, dynamics, and, specifically, T-tubule/SR membrane maintenance in differentiated muscle.

\section{Materials and Methods}

\section{Dogs and Sample Collection}

Homozygote (affected) $(n=5)$ and heterozygote (clinically normal) $(n=2)$ Labrador retrievers (genotyped according to Pelé et $\left.\mathrm{al}^{29}\right)$, in addition to normal golden retrievers $(n=2)$, were selected at the École nationale vétérinaire d'Alfort (Maisons-Alfort, France). Experiments on dogs were approved by the Anses/EnvA/Upec Ethics Committee, and all care and manipulations were performed in accordance with national and European legislation on animal experimentation. Clinical severity varied slightly among the affected dogs, but all had characteristic signs of paresis, reduction in muscle mass, and absent myotatic reflexes typical of the disease. ${ }^{25-29}$ Previous work had determined the progression of general pathologic features in a severely affected muscle, the biceps femoris $^{26-29}$; therefore, this muscle was selected for further evaluation by light and electron microscopy. Biopsy samples were obtained in a standard manner from dogs under general anesthesia. ${ }^{28,29}$ For the evaluation of temporal progression, dogs from a single litter were biopsied twice (from alternate sides) at two ages chosen to give a good representation of pathological features (at 11 months old, during the initial progressive stage of the disease, and again at 30 months of age, when dogs would likely have advanced pathologic features). Additional control samples were obtained from normal beagle dogs $(n=4)$ kept in research colonies within the UK immediately after their euthanasia (for reasons unrelated to this study). Details of all dogs used in this work are summarized in Supplemental Table S1. This article was approved by the Royal Veterinary College's research office.

\section{Muscle Histologic Analysis}

Routine histologic and histochemical stains were used to examine the pathologic features in a subset of samples to 
confirm they were representative. ${ }^{25,27}$ Canine muscle biopsy samples and additional test and control samples were first orientated for sectioning on corks with optimum cooling temperature mountant (Tissue-Tek OCT, Sakura Finetek UK Ltd., Thatcham, UK) and frozen in liquid nitrogen-cooled isopentane. ${ }^{26,28}$ Cryosections $(10-\mu \mathrm{m}$ thickness) from dogs detailed in Supplemental Table S1 were stained with a panel of standard histologic stains: hematoxylin and eosin (H\&E), modified Gomori trichrome, nicotinamide adenine dinucleotide hydride, succinate dehydrogenase, cytochrome oxidase, Oil Red $\mathrm{O}$, and acid phosphatase. The oxidative staining pattern was evaluated using mitochondria-specific stains (cytochrome oxidase and succinate dehydrogenase) in addition to nicotinamide adenine dinucleotide hydride, which also stains SR. ${ }^{1}$ The proportion of centrally nucleated fibers (from a total of $\geq 500$ fibers per dog taken in at least two fields of view) was determined from randomly acquired images obtained using the $10 \times$ objective from $\mathrm{H} \& \mathrm{E}$-stained cryosections using ImageJ software version 1.45g (NIH, Bethesda, MD; http:// imagej.nih.gov/ij). Pathologic abnormalities were assessed [observer (G.L.W.) blinded to genotype] by subjective scoring (performed on H\&E, Gomori trichrome, and cytochrome oxidase, succinate dehydrogenase, and nicotinamide adenine dinucleotide hydride oxidative enzyme stains), and objective counts of myofibers were performed from randomly acquired images (obtained using the $20 \times$ objective) of succinate dehydrogenase-stained sections (minimum of 250 fibers per dog taken on three fields of view or more) (Table 1).

\section{Immunohistochemical Staining}

Immunohistochemistry (IHC) was performed using a selection of antibodies to triad, membrane, cytoskeletal, and myofibrillar proteins based on prominent ultrastructural features (see below) and human CNMs. Use of each new antibody, fixation, and permeabilization protocols was optimized on trial sections in parallel with control human muscle sections and additional canine disease controls (not shown). ${ }^{43}$ IHC was performed by incubating cryosections with each primary antibody in a humidified chamber for 1 hour at room temperature, after rinsing in copious amounts of phosphate-buffered saline, species-appropriate secondary antibodies [goat anti-mouse IgG conjugated to Alexa Fluor 488, goat anti-mouse IgG conjugated to Alexa Fluor 594, and goat anti-rabbit IgG conjugated to Alexa Fluor 594 (Invitrogen, Fisher Scientific UK Ltd., Loughborough, UK)]

Table 1 Severity and Progression of Pathological Features in Dogs with HACD1 CNM at Different Age

\begin{tabular}{|c|c|c|c|c|c|c|c|c|}
\hline Feature & Ctl5 & Ctl6 & CNM1 & CNM2 & CNM1-2 & CNM2-2 & Het2 & CNM5 \\
\hline \multicolumn{9}{|l|}{ Objective counts } \\
\hline $\begin{array}{l}\text { H\&E - centralized nuclei, \% over absolute } \\
\text { count }\end{array}$ & 0.8 & 0.4 & 14.0 & 15.6 & 38.5 & 51.7 & 1.7 & 43.2 \\
\hline \multirow{2}{*}{$\begin{array}{l}\text { SDH - abnormal pattern, \% over absolute } \\
\text { count }\end{array}$} & 0.3 & 0.9 & 78.0 & 81.5 & 85.2 & 86.6 & 7.1 & 90.3 \\
\hline & $1 / 385$ & $3 / 331$ & $199 / 255$ & $296 / 363$ & $225 / 264$ & $219 / 253$ & $22 / 308$ & $261 / 289$ \\
\hline $\begin{array}{l}\text { SDH - halo pattern, \% over absolute } \\
\text { count }\end{array}$ & 0 & 0 & 50.6 & 51.0 & 16.7 & 22.1 & 0 & 6.9 \\
\hline Centralized nuclei & 0 & 0 & \pm & \pm & ++ & ++ & $0 /+$ & ++ \\
\hline Variable myofiber size & 0 & 0 & ++ & +++ & ++ & +++ & \pm & +++ \\
\hline Abnormal internal staining pattern & 0 & 0 & ++ & ++ & $++/+++$ & +++ & $0 /+$ & $++/+++$ \\
\hline Fatty infiltration or myofiber replacement & 0 & 0 & $0 /+$ & $0 /+$ & $++/+++$ & ++ & $0 /+$ & +++ \\
\hline Fibrosis & 0 & 0 & 0 & $0 /+$ & ++ & ++ & $0 /+$ & +++ \\
\hline COX - abnormal pattern & 0 & 0 & ++ & +++ & $++/+++$ & +++ & 0 & +++ \\
\hline $\mathrm{NADH}$ - abnormal pattern & 0 & 0 & $++/+++$ & +++ & +++ & +++ & $0 /+$ & +++ \\
\hline SDH - abnormal pattern & 0 & 0 & ++ & $++/+++$ & +++ & +++ & $0 /+$ & +++ \\
\hline SDH - halo pattern & 0 & 0 & $+/++$ & ++ & \pm & $+/++$ & 0 & \pm \\
\hline \multicolumn{9}{|l|}{ Subjective scoring - other } \\
\hline Oil Red 0 - fat & 0 & 0 & $+/ 0$ & $+/++$ & $++/+++$ & ++ & \pm & +++ \\
\hline Acid phosphatase positive staining result & 0 & 0 & 0 & 0 & 0 & 0 & 0 & 0 \\
\hline
\end{tabular}

Summary of pathologic features as derived from blinded evaluation (G.L.W.) of stained cryosections from each dog. Objective counts were performed to determine the percentage of fibers with centralized nuclei and abnormal oxidative staining pattern from random images of H\&E and SDH stained cryosections, respectively. The lower section of the table gives the results from subjective scoring of other features defined as follows (a range is given where the feature varied across the section): 0 , within normal limits or feature absent; + , mild (on subjective evaluation this feature is apparent but less than a third of fibers or of the field of view is affected); ++ , moderate (approximately half of all fibers or of the field of view is affected); and +++ , severe (greater than two-thirds of fibers or of the field of view is affected).

CNM, centronuclear myopathy; COX, cytochrome oxidase; Ctl, control; H\&E, hematoxylin and eosin; Het2, heterozygote 2; SDH, succinate dehydrogenase. 
were applied at 1:1000 in phosphate-buffered saline for 1 hour at room temperature in a light-proof humidified chamber. Primary antibodies used were mouse monoclonal antibodies, unless otherwise stated: RyR1 [34C (Abcam, Cambridge, UK) 1:100], DHPR [1A (Affinity Bioreagents, Fisher Scientific UK Ltd.) 1:100], sarcoplasmic reticulum Ca2+-ATPase (SERCA2) [IID8 (Affinity Bioreagents) 1:500], dysferlin [1/7B6 (Leica Novocastra, Leica Biosystems, Milton Keynes, UK) 1:25], caveolin 3 [26 (Becton Dickinson, Oxford, UK) 1:200], desmin [D33 (Dako UK Ltd., Ely, UK) 1:100], sarcomeric myosin [MF20 (Developmental Studies Hybridoma Bank, Iowa City, IA) 1:50], developmental myosin heavy chain [RNMy2/9D2 (Leica Novocastra) 1:20], dystrophin [Dys2 (Leica Novocastra) 1:20], rabbit polyclonal antibody against BIN1 (iso 8) (R2406 $\left.{ }^{21} 1: 600\right)$. To obtain double labeling with multiple mouse monoclonal antibodies for evaluation of RyR1 (labeled with Alexa Fluor 594) with DHPR and SERCA2 (labeled with Alexa Fluor 488), Zenon antibody labels were used according to the manufacturer's instructions (Invitrogen, Fisher Scientific UK Ltd.).

Images were obtained using standardized exposures on a Leica DMRA2 wide-field fluorescent microscope (Leica Microsystems, Sunnyvale, CA) equipped with an AxioCam MRm monochrome camera controlled via Axiovision software version 4.8.2 (Carl Zeiss Ltd., Cambridge, UK). Confocal fluorescence microscopy images were captured using a Leica SP5 confocal laser scanning microscope using Argon (488), HeNe (594), and Diode (405) lasers.

\section{Electron Microscopy}

Each muscle sample was kept chilled until placed in fixative within 10 minutes of biopsy. The entire sample (approximately $4-\mathrm{cm}$ long and $1-\mathrm{cm}$ diameter) was initially placed into $4 \%$ glutaraldehyde in $0.1 \mathrm{mmol} / \mathrm{L}$ cacodylate buffer (pH 7.2) (Agar Scientific, Stanstead, UK) while clamped (to preserve myofiber length). The sample was then dissected further while in fixative (typically within 10 minutes) to produce small blocks ( 1 to $2 \times 2$ to $4 \mathrm{~mm}$ ) oriented with the long axis running along the myofibers; these samples then remained in fixative for 2 hours at room temperature. Samples were then rinsed in a single wash of $0.1 \mathrm{mmol} / \mathrm{L}$ cacodylate buffer and stored at $4^{\circ} \mathrm{C}$ until further processing. Subsequently, samples were processed in a standard manner using a mechanical tissue processor (Lynx EL EM Microscopy Tissue Processor, Reichert Jung, Ametek, Munich, Germany). Tissue was rinsed in $0.1 \mathrm{mmol} / \mathrm{L}$ cacodylate buffer, postfixed in $1 \%$ osmium tetroxide (Agar Scientific) for 2 hours at $4{ }^{\circ} \mathrm{C}$, dehydrated through graded ethanols and propylene oxide, and embedded in Araldite resin (Agar Scientific) for both transverse and longitudinal sectioning. Blocks were prepared and semithin sections (500 to $800 \mathrm{~nm}$ ) cut and stained with toluidine blue $(1 \%$ saturated with borax, TAAB Laboratories Equipment Ltd., Aldermaston, UK) to select blocks in the desired orientation. Ultrathin sections (approximately 65-nm thickness) were prepared from each dog. Sections for imaging were stained with saturated methanolic uranyl acetate and Reynold's lead citrate and examined on a CM10 transmission electron microscope (Philips, FEI UK Ltd., Cambridge, UK). Images were obtained using a Kodak Megaview III camera (SIS $\mathrm{GmbH}$, Munster, Germany).

\section{Imaging, Measurement, and Statistical Analysis of T-Tubule Morphometry}

T-tubules were evaluated morphometrically from electron micrographs to provide quantitative, objective data. Images used for T-tubule measurements were captured from longitudinal sections at $\times 21,000$ magnification in an identical manner for all samples. Evaluations of shape and size were performed for $>120$ T-tubules per dog from a minimum of five myofibers (Supplemental Table S1). T-tubules in the images were circumscribed using a pen tablet (intuos4, Wacom Europe GmbH, Krefeld, Germany), and parameters describing shape (circularity, as evaluated by Cowling et $\mathrm{al}^{16}$ ) and size were measured for each T-tubule using ImageJ software. Values were exported into Microsoft Excel version 14.3.9 (Microsoft Inc., Redmond, WA) and Prism software version 6.0 (GraphPad Software Inc., La Jolla, CA) for further analysis and graphical representation. Statistical analysis, by mixed-effect modeling, was performed with SPSS software version 2.0.3 (IBM, Portsmouth, UK).

\section{Cell Lines and Tissue Culture}

A clonal $\mathrm{C} 2 \mathrm{C} 12$ myoblast cell line, stably transfected with pGIPZ shRNA construct -V2KLHS_5923 (GCTCATTACTCACAGTATA), Thermo Fisher Scientific, Waltham, MA] against exon 4 of Hacd1, was evaluated along with an additional clone expressing a control plasmid (RHS4349). ${ }^{40}$ These were grown and differentiated in media that contained $2 \mu \mathrm{g} / \mathrm{mL}$ of puromycin to select for stable transgene expression in the pGIPZ transfected cells. Cultures were incubated at $37^{\circ} \mathrm{C}$ in $5 \% \mathrm{CO}_{2}$. Myoblasts were grown in Dulbecco's modified Eagle's medium (Sigma-Aldrich Company Ltd., Dorset, UK) containing 10\% heat inactivated fetal bovine serum (PAA Laboratories, Linz, Austria), $2 \mathrm{mmol} / \mathrm{L}$ L-glutamine (Invitrogen), $100 \mathrm{IU} / \mathrm{mL}$ of penicillin, and $0.1 \mathrm{mg} / \mathrm{mL}$ of streptomycin (Sigma-Aldrich Company Ltd.). Cells were plated into tissue culture flasks (Nunc, Thermo Fisher Scientific) or hydrophilic-coated optical-bottomed dishes (ibiTreat-coated $\mu$-dishes, Thistle Scientific, Glasgow, UK) for immunocytochemistry and differentiated at $70 \%$ to $80 \%$ confluence in Dulbecco's modified Eagle's medium that contained $4 \%$ heatinactivated horse serum (PAA Laboratories) and L-glutamine, penicillin, and streptomycin as above. In these conditions, a partial rescue of the phenotype previously reported $^{40}$ enabled this line of Hacd1 knockdown (KD) myoblasts to form myotubes. 
Quantitative RT-PCR was used to compare expression of the skeletal muscle-specific Hacdl transcript between the control and HACD1-deficient cells. Cells were plated into flasks and harvested as myoblasts and at 2, 7, and 14 days' differentiation. Relative expression of Hacd 1 compared with Gapdh was evaluated using a modified $\Delta \mathrm{C}_{\mathrm{T}}$ method with efficiency correction. ${ }^{44}$ Primer pairs specifically amplified the skeletal muscle Hacd1 full-length isoform (forward: $5^{\prime}$ ATGAAGAGAGCGTGGTGCTT-3'; reverse: 5'-AAGGCGGCGTATATTGTGAG-3') for comparison with a housekeeping gene (Gapdh) (forward: 5'-TTGTGATGGGTGTGAACCAC-3'; reverse: 5'-TTCAGCTCTGGGATGACCTT-3' 11) that has constant expression during myoblast differentiation. ${ }^{45}$ Significant Hacd1 KD (approximately $80 \%$ ) was found between the Hacd1 KD and shRNA plasmid control cell lines at all time points.

\section{Immunocytochemistry and Cell Microscopy}

Cells plated into optical dishes (ibiTreat-coated $\mu$-dishes) were rinsed in phosphate-buffered saline and fixed and permeabilized in methanol and acetone (50:50) for $4 \mathrm{mi}-$ nutes at $-20^{\circ} \mathrm{C}$ (those to be stored before staining were allowed to air dry and then kept at $-20^{\circ} \mathrm{C}$ ). Immunostaining was performed using primary and secondary antibodies as described for muscle cryosections except nuclei that were labeled with [Hoechst 33342 (Invitrogen) 1:5000 for 5 minutes at room temperature] before mounting with Hydromount (Agar Scientific) using a 19-mm diameter coverslip (VWR, Lutterworth, UK).

Myotube morphologic features were evaluated (G.L.W., blinded to type) by manual counting performed from two independent experiments per cell line, which generated eight random $10 \times$ field-of-view images from standard areas of RyR1-stained cells at 12 days' differentiation. Significance was evaluated by Fisher exact test (GraphPad Software Inc.).

\section{Results}

Histopathologic features of the dogs used in this study and representative of the disease are depicted in Figure 1 and Table 1. On H\&E-stained cryosections, abnormal features of central nucleation, variability in myofiber size, fibrosis and myofiber loss, and replacement by fat infiltration were seen in affected HACDl CNM dogs. Variability in myofiber size with large numbers of small hypotrophic fibers were prominent from the earlier time point (Figure 1D), whereas other features, including the proportion of internalized nuclei, became more apparent with increasing age (Figure 1, G, J, and M, and Table 1). In healthy dogs, oxidative stains have a regular distribution on transverse section within a myofiber, and intensity varies based on fiber type between myofibers (type 1 darker staining) (Figure 1B). Abnormal oxidative staining patterns observed in affected dogs included the absence of a clear fiber-type distribution and the presence of necklace fibers with an internal, dense-staining ring, running parallel to the sarcolemma, lobulated fibers with dense subsarcolemmal mitochondrial deposits and many fibers with a halo or peripheral rim devoid of oxidative staining (Figure 1, E, H, K, and N). Blinded, subjective scoring (G.L.W.) revealed that most fibers from HACDI CNM dogs of all ages had abnormal internal cytoarchitecture with histologic and oxidative stains (Table 1). The presence of a peripheral halo was a particularly prominent feature in 11-month-old dogs (present in approximately $50 \%$ of all fibers) (Figure $1 \mathrm{~N}$ ); in 30-monthold dogs, this pattern was superseded by more generalized disorganization. Presumed secondary degenerative changes (fat infiltration and fibrosis) were more common in older compared with younger dogs (Figure $1, \mathrm{G}$ and $\mathrm{J}$, and Table 1). Ultrastructural abnormalities observed in affected dogs with HACD1 CNM included internalized or centralized nuclei (Figure 1, F, I, and L), mitochondrial mislocalization and clumping (Figure 1F), presence of lipid bodies (Figure 1, I and L), and myofibrillar disorganization (Figure 1, I and L). These features appeared most severe in the older animals. No significant abnormalities were detected in the heterozygous or control dogs (Figure 1, A-C). Prominent features observed by electron microscopy were probed further by IHC on cryosections from the same biopsy samples (see below).

\section{Progressive Triad Abnormalities in HACD1-Deficient Canine CNMs}

Recent studies have implicated defective excitationcontraction coupling in the pathogenesis of CNMs linked to mutations in genes other than HACDI and have documented T-tubule abnormalities as a consistent feature in human patients and animal models. ${ }^{11-14,16,18-21}$ Consequently, to support a link between canine $H A C D I C N M$ and other CNM forms, we evaluated the effect of the HACDI mutation on muscle membranes in more detail.

Initial subjective evaluation of muscle ultrastructure revealed a proportion of T-tubules that appeared abnormally rounded and dilated in affected dogs (Figure 2, A-D). Objective morphometric evaluation of shape and size revealed that normal T-tubules were elliptical [means of medians \pm SD for circularity, $0.499 \pm 0.033$ arbitrary units $(\mathrm{AU})]$ with uniform cross-sectional area $(0.0021 \pm 0.0003$ $\mu^{2}$ ). In HACDI CNM 11-month-old dogs, circularity and variability were increased $(0.755 \pm 0.045 \mathrm{AU} ; P<0.001)$ with an insignificant increase in area $\left(0.0026 \pm 0.0005 \mu \mathrm{m}^{2}\right.$; $P=0.086)$. At 30 months of age, both circularity $(0.739 \pm 0.051 \mathrm{AU} ; P<0.001)$ and cross-sectional area $\left(0.0051 \pm 0.0008 \mu \mathrm{m}^{2} ; P<0.001\right)$ were significantly increased (Figure 2, E and F, and Supplemental Figure S1). In 30-month-old dogs, additional membranous abnormalities were observed, including dilated T-tubules with luminal contents (Figure 2C). 

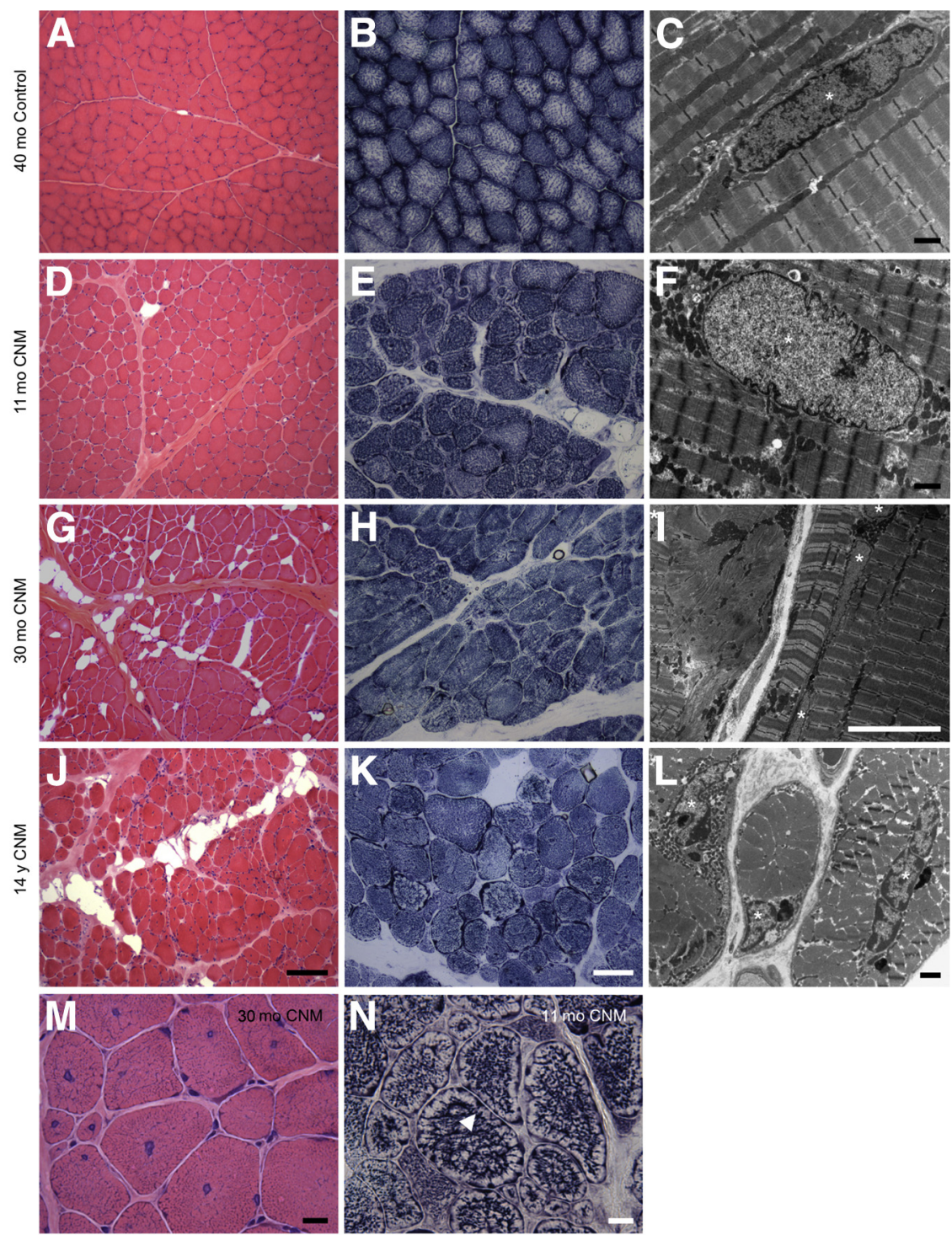

Figure 1 Typical pathologic features of $H A C D 1$ centronuclear myopathy (CNM) in Labrador retriever dogs. A, D, G, and J: Representative lowmagnification images of hematoxylin and eosinstained $10-\mu \mathrm{m}$ cryosections from biceps femoris muscle biopsy samples from a control 40-month-old $\operatorname{dog}(\mathbf{A})$ and HACD1 CNM affected dogs at 11 months old (D), 30 months old (G), and 14 years old (J). Note the variability in myofiber size and greater fibrosis and fat infiltration in the older CNM-affected animals. M: At higher magnification, from a $30-$ month-old affected dog in whom prominent pathologic features, such as abnormal internal staining pattern, centralized nuclei, and small hypotrophic fibers, are evident. B, E, H, and K: Succinate dehydrogenase histochemistry of $10-\mu \mathrm{m}$ cryosections from biceps femoris muscle biopsy samples from a control adult (B) and HACD1 CNM affected dogs at 11 months old (E), 30 months old $(\mathbf{H})$, and 14 years old (K). Note the loss of fiber-type checkerboard pattern and abnormal pattern of oxidative staining. $\mathrm{N}$ : Higher-magnification image is from an 11month-old CNM-affected dog and shows numerous myofibers with the halo pattern (arrowhead) that was a common feature. C, F, I, and L: Lowmagnification muscle ultrastructure showing representative features in affected dogs at 11 months old (F), 30 months old (I), and 14 years old (L) compared with a control adult dog (C). C: Normal myonuclei (asterisk) are peripherally located, whereas in HACD1 CNM-affected dogs, abnormal internalization can be seen. These were often associated with clumps of mitochondria and, particularly in older dogs, clumps of dark staining amorphous material (lipid bodies). I: Also notable in the 30-month-old dog is an area of focal disorganization at the myofiber periphery. In addition, internalized myonuclei can be seen in a chain (asterisks). Scale bars: $100 \mu \mathrm{m}(\mathbf{A}, \mathbf{D}, \mathbf{G}$, and J); $50 \mu \mathrm{m}$ $(\mathbf{B}, \mathbf{E}, \mathbf{H}$, and $\mathbf{K}) ; 10 \mu \mathrm{m}(\mathbf{M}, \mathbf{N}$, and $\mathbf{I}) ; 1 \mu \mathrm{m}(\mathbf{C}, \mathbf{F}$, and $\mathbf{L})$.

\section{Disorganization of T-Tubule and SR Membrane Markers, Including BIN1 and RyR1}

To evaluate the distribution of triads in muscle from affected dogs, immunostaining for T-tubule (DHPR) and SR (SERCA and RyR1) markers was performed on transverse cryosections from the same biopsy samples used for electron microscopy. RyR1 and DHPR were observed broadly to colocalize, and triad staining was disorganized in $H A C D 1$ CNM dogs at both ages. In control dogs, a normal, regular, honeycomb pattern could be seen outlining the bundles of myofilaments (Figure 3A). In affected dogs, the staining was disorganized and density was reduced at the periphery, except for radial remnants directed toward the sarcolemma (Figure 3, B and C). At 30 months of age, focal areas of RyR1 and DHPR expression were associated with the periphery of internalized nuclei or were distributed within the sarcoplasm, parallel to the sarcolemma (Figure 3C and Supplemental Figure S2).
BIN1, a T-tubule associated protein with a critical role in membrane tubulation and T-tubule development ${ }^{46}$ and recruitment of partner proteins, ${ }^{47-49}$ has disorganized localization in several human CNMs, irrespective of genotype. ${ }^{18}$ Double labeling, with antibodies directed against BIN1 and RyR1 (Figure 3, D-F, and Supplemental Figure S3) revealed a similar pattern of disorganization as seen with DHPR and RyR1 triad staining. Generally, BIN1 and RyR1 colocalized except at the nuclear periphery; some aggregates were positive for BIN1 but not RyR1. In longitudinal sections, areas of intense BIN1 and DHPR labeling extended from the centralized nucleus (Figure 3, G-J). On reevaluation of the muscle ultrastructure in light of these findings, the prominent subsarcolemmal tubular and vesicular membranous structures tended toward a similar radial alignment and were considered likely to originate from T-tubule and SR networks (Figure 3, K and L).

Thus, dogs with HACDI CNM have alterations in T-tubule ultrastructure, including altered shape and 

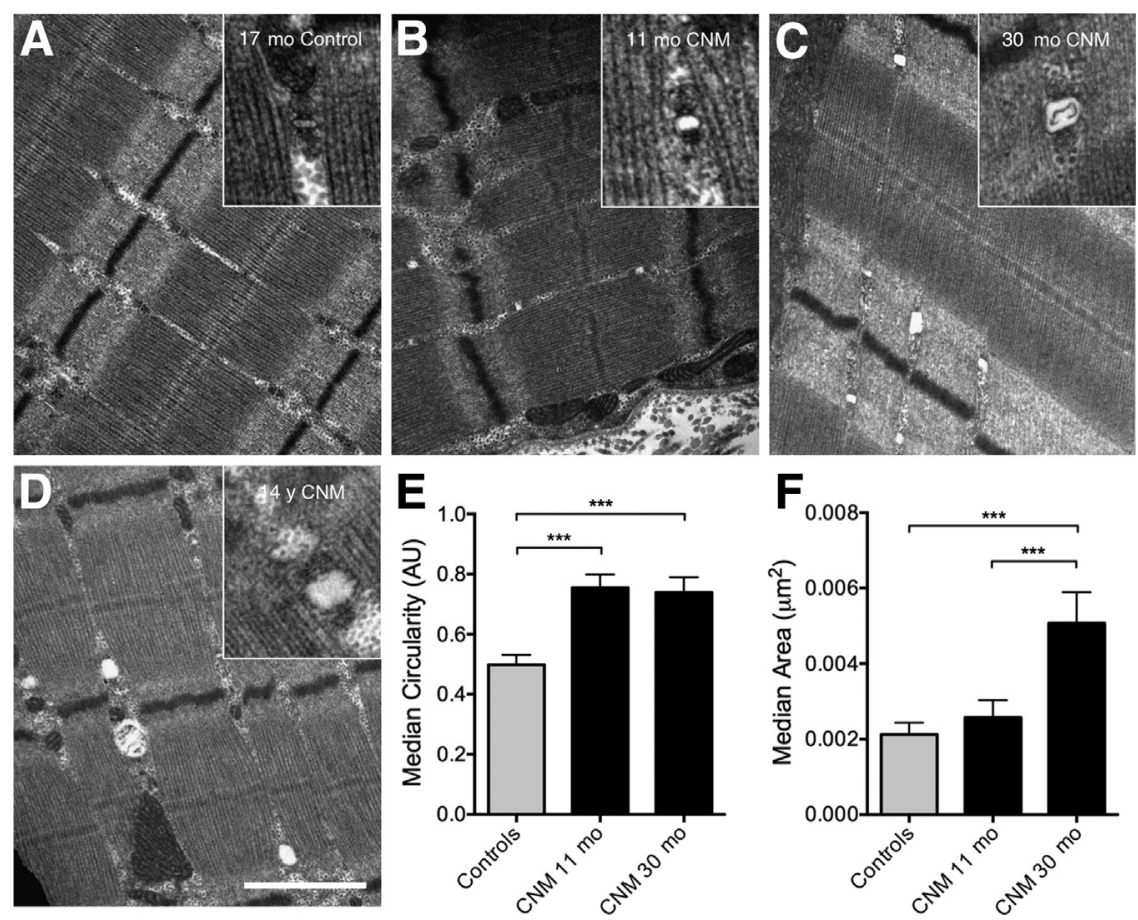

Figure 2 Triad abnormalities in dogs with canine HACD1 centronuclear myopathy (CNM). AD: Electron micrographs from longitudinal biceps femoris muscle sections showing T-tubule appearance from normal canine muscle (17-month-old $\operatorname{dog})(\mathbf{A})$ and affected dogs at 11 months old (B), 30 months old (C), and 14 years old (D). Insets show selected representative single T-tubules in more detail. Note the narrow, elliptical T-tubules in the normal dog, whereas in dogs with HACD1 CNM, T-tubules appear more rounded and more dilated as they age. The inset in C shows a Ttubule with membranous contents. $\mathbf{E}$ and $\mathbf{F}$ : Results of objective measurements of T-tubule morphometry performed in $>125$ T-tubules per dog from at least five myofibers: circularity $(\mathbf{E})$ and area $(\mathbf{F})$ are depicted in bar graphs. A mixed-effect model was used to assess significance; compared with control dogs, circularity was increased in affected dogs at both time points, and area was significantly increased at the later time point. Supplemental Figure S1 shows these results in detail with all data points plotted for each dog. Data are expressed as means of medians \pm SD (E and $\mathbf{F})$. ${ }^{* * *} P<0.001$. Scale bars: $1 \mu \mathrm{m}$ (main images); $0.5 \mu \mathrm{m}$ (insets). $\mathrm{AU}$, arbitrary units. progressive dilation. Triad distribution is increasingly disorganized, with mislocalization especially from areas close to the sarcolemma.

\section{Mitochondrial Mislocalization and Alterations in Membrane Trafficking and Repair-Associated Proteins in Affected Dogs}

Mitochondrial mislocalization and degeneration (as revealed by clumping and whorls) were associated with areas of suspected tubuloreticular membranous abnormalities (Figure 3, K and $\mathrm{L}$ ). In addition, the peripheral disruption noted on triad staining appeared analogous to that of the halo pattern detected with oxidative histochemistry, and this correlation was confirmed using serial sections (Figure 4, A-G). SERCA2 (Figure 4, G-I, and Supplemental Figure S4) was used as an additional SR marker that specifically labels the calciumATPase in type 1 myofibers. This otherwise colocalized with RyR1, and despite the few remaining type 2 fibers, both fiber types had the same pattern of SR disorganization.

Electron microscopy revealed the presence of sarcolemmal abnormalities, including membrane invaginations, and subsarcolemmal membranous structures, including vesicles and caveolae (Figure 5, A-D). In 11-month-old dogs, dysferlin was mislocalized in the cytoplasm in a number of fibers, diffusely or revealing a peripheral halo (Figure 5, F and G). Developmental myosin immunolabeling on serial sections revealed that a small proportion of intensely stained fibers with cytoplasmic mislocalization of dysferlin were also positive for this marker of regeneration or immaturity (Figure 5H) (commercially available antibodies for embryonic and neonatal myosins do not detect canine isoforms ${ }^{50}$ ). Adult dogs also had cytoplasmic dysferlin expression (Figure 5I) and an additional pattern, also seen with dystrophin, which suggested the presence of invaginated or internalized sarcolemmal membranes (Figure 5, J-L). Caveolin 3 had a normal distribution in 11month-old dogs but had similar abnormalities to dysferlin in adults (30-month-old and 14-year-old dogs) (results not shown).

\section{Cytoskeletal Disorganization}

In addition to the membranous defects described above, we also observed more generalized disorganization affecting cytoskeletal and contractile elements. Z-line abnormalities, including accumulations and rods, and myofibrillar disorganization were detected (Figure 6, A-F), and the intermediate filament protein, desmin (Figure 6, G-I), and sarcomeric myosin (Figure 6, J-L) were mislocalized.

\section{Altered Cellular and Membrane Morphologic Findings in HACD1-Deficient C2C12 Myotubes}

We previously found that myoblast fusion is supported by HACD1 expression. ${ }^{40}$ The differentiation conditions used in this study enabled a partial rescue of the phenotype, allowing us to maintain sarcomeric myosin-positive HACD1-deficient myotubes until 12 days of differentiation (Supplemental Figure S5). Both control and Hacd1 KD lines formed RyR1-positive myotubes (Figure 7, B and E), but myotubes from the HACD1-deficient line were observed with intracellular vesicles (Figure 7, A and D), and many had unusual clusters of nuclei that formed complete or partial rings (either in a rounded, multinucleate cell or an 

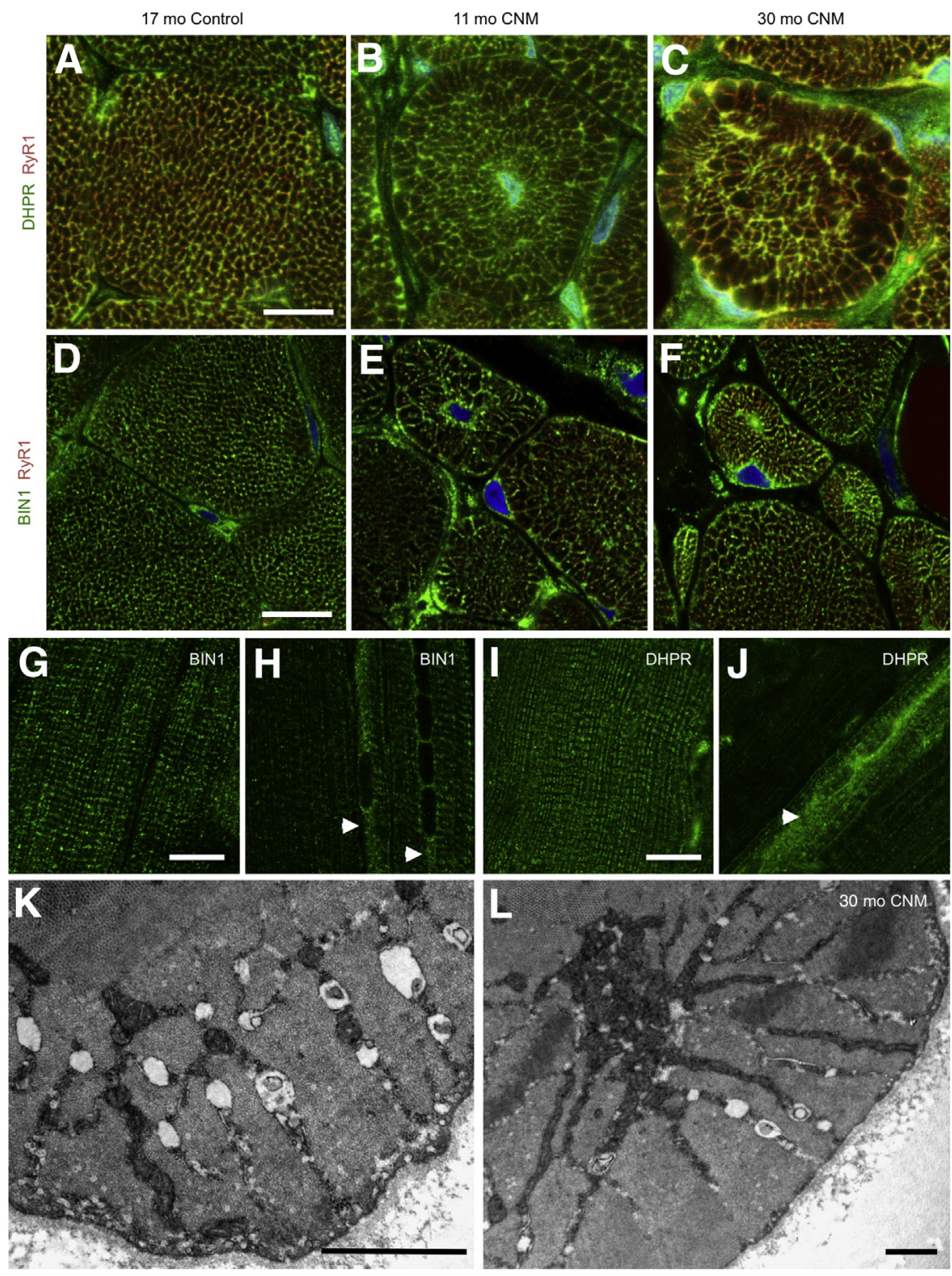

Figure 3 Triad immunohistochemistry. A-F: Confocal images of transverse sections stained for dihyropyridine receptor (DHPR) and ryanodine receptor (RyR1) (A-C) and amphiphysin 2 (BIN1) and RyR1 (D-F). High-magnification images of representative myofibers from 17-month-old control (A and D), 11-month-old centronuclear myopathy (CNM) (B and E), and 30-month-old CNM (C and F) canine muscle. Note the regular honeycomb pattern of the staining that outlines the bundles of myofilaments in the healthy dog that is disrupted in the affected dogs with loss of this pattern and reduced staining intensity, particularly in the fiber periphery. In the older affected dog, this becomes more prominent with more general disorganization and focal areas of dense staining. DHPR and RyR appear to colocalize variably across the fiber. $\mathbf{G}-\mathbf{J}$ : Confocal images of longitudinal cryosections of biceps femoris muscle from 40-month-old control ( $G$ and $\mathbf{I}$ ) and 11-month-old CNM-affected ( $\mathbf{H}$ and $\mathbf{J}$ ) dogs stained for BIN1 ( $\mathbf{G}$ and $\mathbf{H}$ ) and DHPR (I and $\mathbf{J})$. Note the accumulations of these T-tubule associated proteins that extend from internalized nuclei (arrowheads). Transmission electron micrograph images of transverse sections showing membranous structures of suspected tubuloreticular origin in 30-month-old CNM affected dogs. $\mathbf{K}$ and $\mathbf{L}$ : The images show varying configurations, including a subsarcolemmal rim of vesicular structures and mitochondria $(\mathbf{K})$ and disorganization with mitochondria and tubular and vesicular membranes with a radial alignment toward the sarcolemma (L). Scale bars: $1 \mu \mathrm{m}(\mathbf{A}-\mathbf{J}) ; 10 \mu \mathrm{m}$ ( $\mathrm{K}$ and $\mathrm{L}$ ). area continuous with a more elongated myotube) (Figure 7, D-F). These clusters of abnormally positioned myonuclei were associated with an area of intense RyR1 staining (Figure 7E). A significantly higher proportion of Hacd1 KD cells had these unusual morphologic findings compared with controls (25 of 147 myotubes versus 3 of 229; $P<0.0001$ ). The skeletal muscle-specific BIN1 isoform (iso 8) had similar localization to that of RyR1: control myotubes were stained relatively homogeneously (Figure 7C), whereas the Hacd1 KD cells seemed to have focal areas of intense staining, often associated with clusters of nuclei (Figure 7F).

\section{Discussion}

Canine CNM, a highly prevalent, naturally occurring, recessive disorder in Labrador retrievers, is characterized by progressive paresis, absent myotatic reflexes, reduced skeletal muscle mass, and histopathologic features suggestive of a CNM, but the pathophysiology has until now been unclear. In particular, the causative HACDI mutation's effect on muscle ultrastructure and subcellular membrane systems, which are implicated in CNMs with different genetic causes, has not previously been investigated. Our work reveals a fundamental role for $H A C D l$ in the maintenance of these structures in skeletal muscle. Furthermore, we found that this large animal model has numerous pathologic features that are closely related to those in human CNMs and smaller laboratory animal CNM models. Our work helps explain the progressive paresis found in affected dogs and provides mechanistic insight into a rare but related human congenital myopathy with closely related clinical features. $^{32}$ 

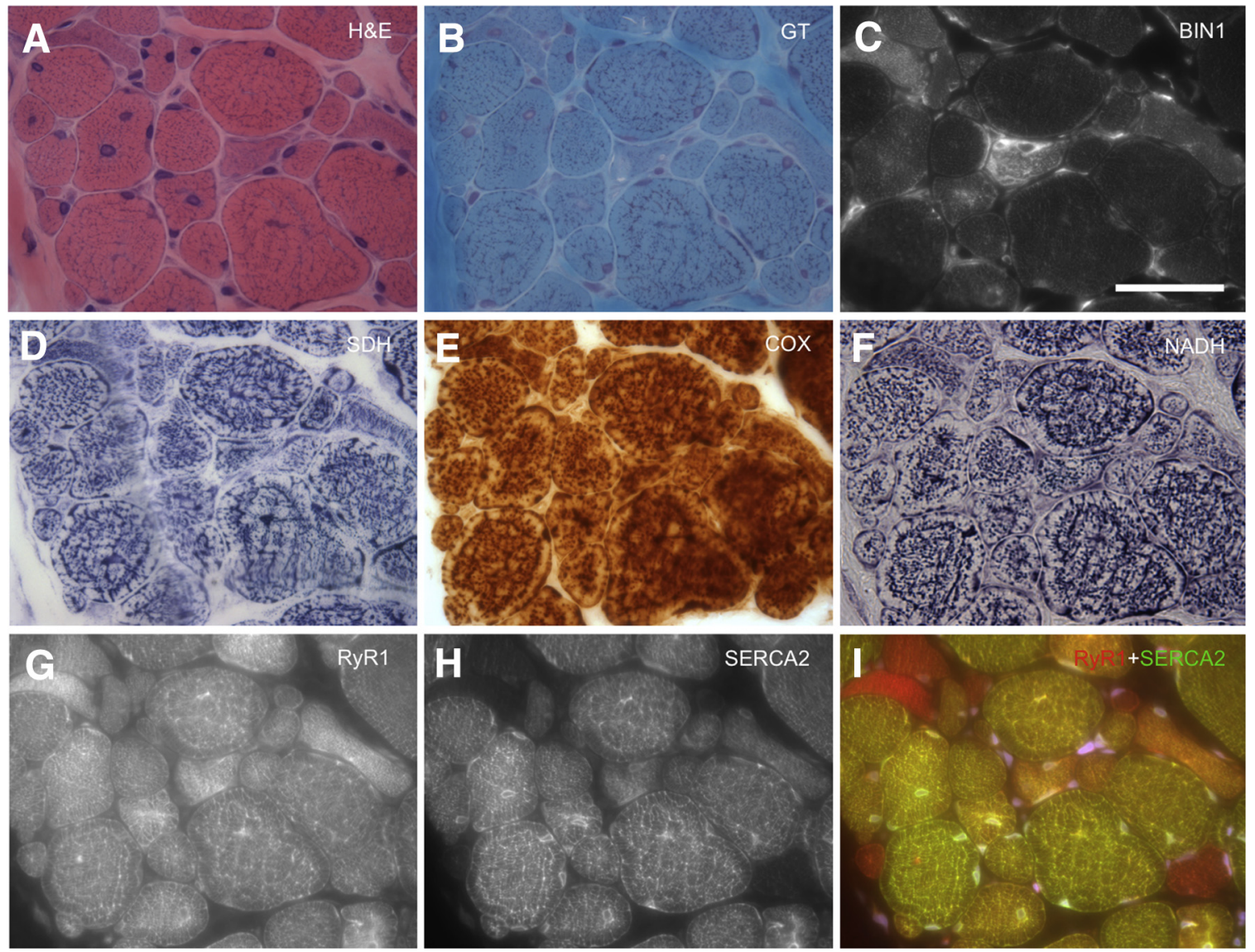

Figure 4 Disorganized tubuloreticular membranes and mitochondria mislocalization. Serial biceps femoris muscle cryosections from an 11-month-old centronuclear myopathy (CNM) affected dog stained with hematoxylin and eosin (H\&E) (A), Gomori trichrome (GT) (B), amphiphysin 2 (BIN1) immunohistochemistry $(\mathbf{C})$, succinate dehydrogenase stains $(\mathrm{SDH})(\mathbf{D})$, cytochrome oxidase $(\mathrm{COX})(\mathbf{E})$, and nicotinamide adenine dinucleotide hydride (NADH-TR) (F) and dual labeling for ryanodine receptor (RyR1) (G) and sarcoplasmic reticulum Ca2+-ATPase (SERCA2) (H) merged with DAPI (I). C-H: These serial sections reveal that disorganization of tubuloreticular membranes $(\mathbf{C}, \mathbf{G}$, and $\mathbf{H})$ corresponds with the appearance of peripheral fiber oxidative halos (D-F). Note that SERCA2 preferentially stains longitudinal sarcoplasmic reticulum in type 1 myofibers; therefore, the type 2 fibers (which are reduced in number in HACD1 CNM affected dogs) are stained with RyR1 alone and appear red in the merged image (I). Representative control images for comparison can be viewed in other figures as follows: H\&E and SDH oxidative staining (Figure 1), RyR1, dihyropyridine receptor, and BIN1 (Figure 3). Scale bar $=50 \mu \mathrm{m}$.

\section{Canine HACD1 Deficiency and Triad Abnormalities Link to Proposed Pathogenesis for Other CNMs}

In skeletal muscle, numerous highly specialized membrane systems interact to convey neuronal input via the neuromuscular junction, conduct these signals internally via T-tubules, and store and release calcium to activate the contractile apparatus and transmit the generated force to the extracellular matrix. Since 2009, T-tubule abnormalities have been described consistently in human patients or animal models with defects in CNM-associated genes ${ }^{15}$ : MTM1, ${ }^{11-13}$ BIN1, ${ }^{17,18,21,51}$ DNM2, ${ }^{14,16,18}$ hJUMPY, ${ }^{19,52}$ and RYRI. ${ }^{22,53}$ In our work, T-tubules from HACD1deficient CNM dogs at both ages were more circular in transverse sections at triads when compared with controls and became significantly larger by 30 months of age. The initial alteration in shape and subsequent dilation suggest that T-tubules remodel or undergo degradative morphologic changes in dogs affected by HACDl CNM. Our identification of apparent membranous contents within the lumen of some T-tubules has not been reported in other myopathies to our knowledge. IHC revealed triad-associated proteins, DHPR and RyR1, were disorganized and depleted from the myofiber periphery. Our results reveal that, as for other CNM-associated genes, including $M T M 1, D N M 2$, and $B I N I,{ }^{11,12,14,21,54}$ HACDI has a functional role in the maintenance of T-tubule morphologic features and localization, either directly or indirectly.

This current study extensively characterized T-tubule morphologic features in $H A C D 1$ CNM; dilated junctional SR terminals were also documented with prominent membranous structures in the intermyofibrillar space and within myofibrillar bundles that might represent aberrant SR membranes (Figures 5, C and D, and 6, D and E). These abnormalities were probed further by IHC to examine the T-tubule system and SR distribution, organization, and physical coupling. Markers for junctional and longitudinal SR (RyR1 and SERCA2, respectively ${ }^{55}$ ) were disorganized, 

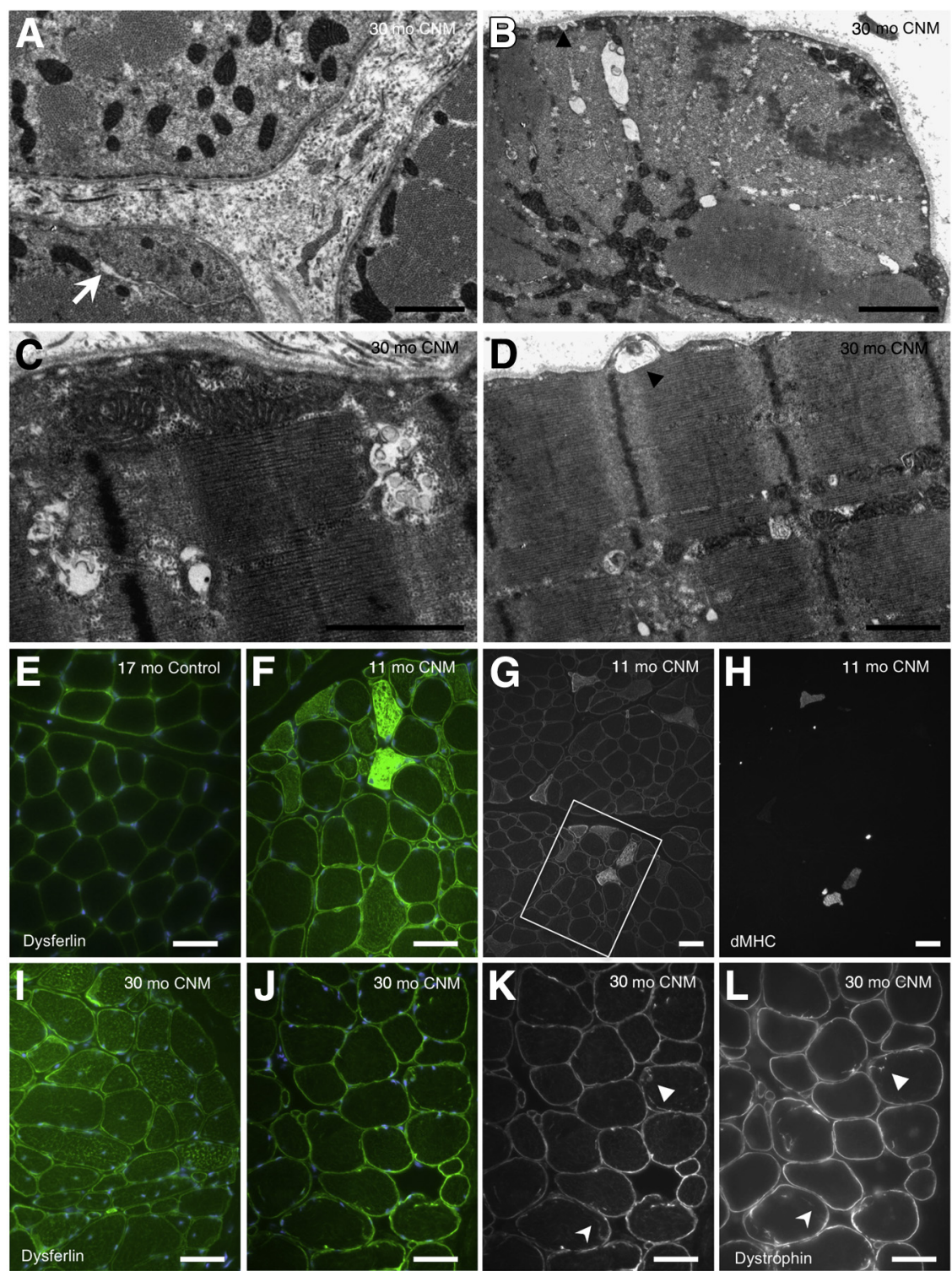

Figure 5 Sarcolemmal and internal membranous abnormalities with dysferlin mislocalization. $\mathbf{A}-\mathbf{D}$ : Transverse ( $\mathbf{A}$ and $\mathbf{B}$ ) and longitudinal ( $\mathbf{C}$ and D) electron micrographs from 30-month-old HACD1 centronuclear myopathy (CNM) affected dogs showing aberrant tubular and vesicular membranous structures, including sarcolemmal membrane invaginations (white arrow) and subsarcolemmal vacuoles and caveolae (black arrowheads). E-J: Dysferlin immunohistochemistry (green) merged with DAPI (blue) on transverse cryosections from control 17-month-old (E), 11-month-old CNM (F), 30-month-old CNM with internal cytoplasmic staining (I), and 30-month-old CNM with internalized focal membranous staining (J). $\mathbf{G}-\mathbf{H}$ : The boxed area in $\mathbf{G}$ shows the region magnified in image $\mathbf{F}$, which corresponds to a serial section stained with developmental myosin heavy chain $(\mathrm{dMHC})(\mathbf{H})$, demonstrating that only a few fibers with diffuse cytoplasmic staining for dysferlin were $\mathrm{dMHC}$ positive (presumably regenerating) fibers. $\mathbf{K}$ and $\mathbf{L}$ : $\mathbf{K}$ shows dysferlin alone (from $\mathbf{J}$ ) alongside a serial section stained with dystrophin (L), revealing that internal membranous elements costain with this subsarcolemmal protein (arrowheads). Scale bars: $1 \mu \mathrm{m}(\mathbf{A}-\mathbf{D}) ; 50 \mu \mathrm{m}(\mathbf{E}-\mathbf{L})$. as were proteins localized to T-tubules $\left(\mathrm{DHPR}^{55}\right.$ and $\mathrm{BIN}^{56}$ ). SERCA2 immunostaining also enabled identification of type 1 myofibers, and although there is a marked shift toward oxidative fibers in this condition, SR defects were identified in both fiber types. RyR1, DHPR, and BIN1 aggregates, similar to those reported here, have been detected previously in canine models of X-linked MTM $^{13}$ and BIN1 autosomal recessive CNM. ${ }^{18,21}$ A recent report localized MTM1 to junctional SR and concluded that altered SR remodeling is likely to be a primary cause of intracellular membrane disorganization in myotubular myopathy. ${ }^{20}$ BIN1 localizes to T-tubules and has a role in their formation. $^{46,47}$ HACD1 is believed to reside with other components of the elongase complex at endoplasmic reticulum or SR membranes ${ }^{34,40}$; however, the localization and specific function(s) of VLCFA and related lipids in muscle remain to be fully characterized. It is not surprising, given the intimate association between tubuloreticular membrane networks, that defects in one will affect the other, producing similar triad abnormalities.

We have also provided evidence of similar membranous abnormalities in Hacd1 KD C2C12 myotubes, which have intracellular vacuoles visible by light microscopy and accumulations of RyR1- and BIN1-positive structures in conjunction with abnormally positioned nuclei. Although this intermediate (myotube) developmental stage in $\mathrm{C} 2 \mathrm{C} 12$ cells precedes organized tubuloreticular networks, rudimentary structures are present. ${ }^{46,57-59}$ These results link HACD1 deficiency in dogs with CNM and a cell culture model to abnormal development and maintenance of triad membranes and BIN1 mislocalization. These are consistent features currently considered to be of primary importance in the pathogenesis of $\mathrm{CNMs}^{15,18}$ and, via postulated dysfunction of excitation-contraction coupling, provide a 

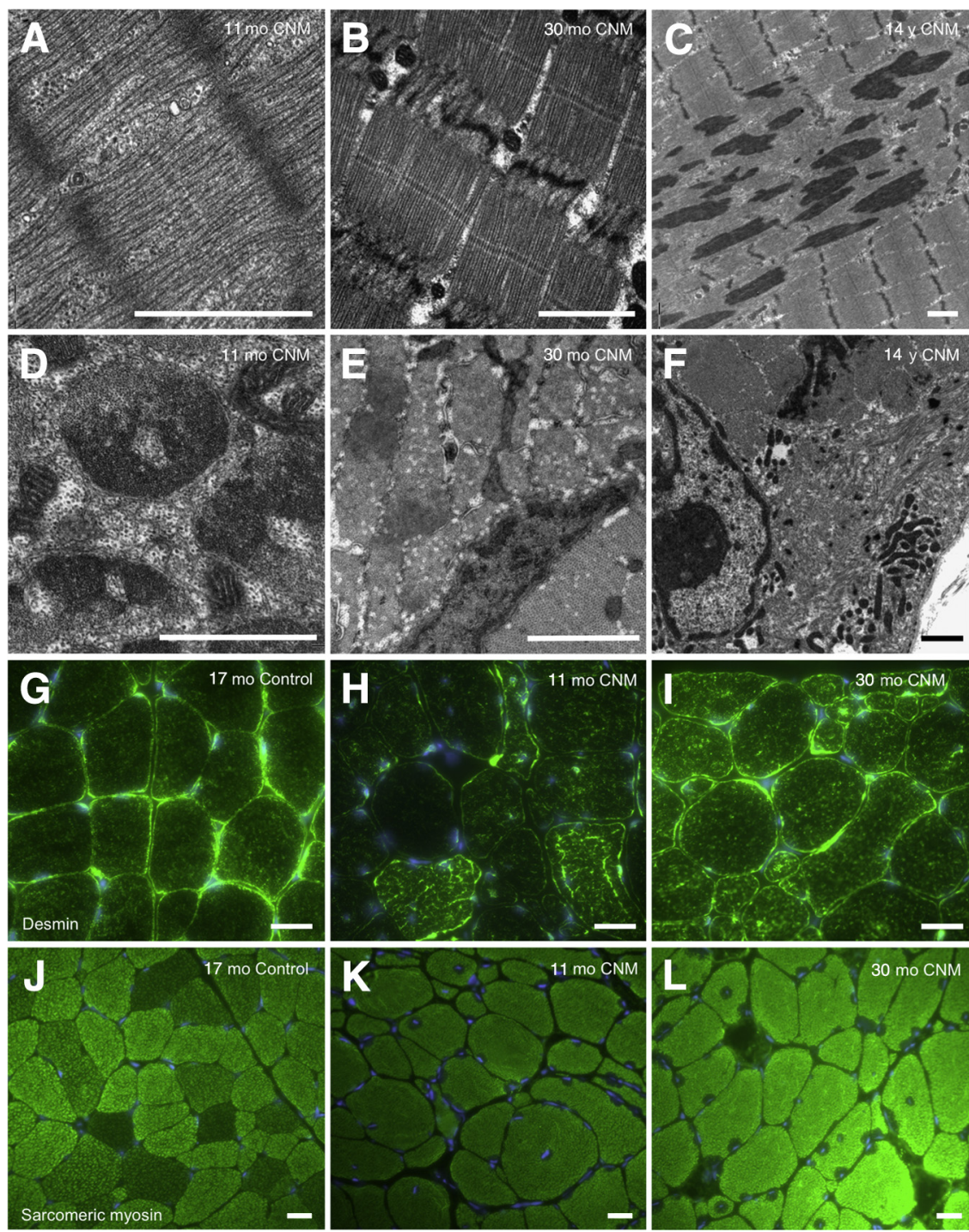

Figure 6 Cytoskeletal abnormalities. A-C: Ultrastructural z-line abnormalities on longitudinal sections. A and B: Hazy and disorganized or smeared z-lines from 11-month-old and 30-monthold HACD1 centronuclear myopathy (CNM) affected dogs, respectively C: Accumulations of z-line material (rods) in a 14-year-old affected dog (these were also observed in 30-month-old dogs). D-F: Myofibrillar disorganization on transverse sections from 11-month-old (D) and 30-month-old (E) CNM affected dogs: intermyofibrillar spaces are widened and bundles of myofilaments disrupted with accumulations of tubular membranes and glycogen. F: Transverse myofiber section from a 14-year-old dog revealing the progression into old age, including a cap of extensive disorganization. $\mathbf{G}-\mathbf{L}$ : Immunohistochemistry for desmin (G-I) and sarcomeric myosin (MF20) (J-L) in 17-month-old control ( $\mathbf{G}$ and $\mathbf{J})$, 11-month-old CNM ( $\mathbf{H}$ and $\mathbf{K}$ ), and 30-month-old CNM (I and $\mathbf{L}$ ) dogs. Note the disorganization of these intermediate filament and contractile apparatus proteins particularly around the nuclei in CNM-affected dogs. Exposures are not the same for desmin in all dogs: affected dogs required shorter exposure times versus normal. Scale bars: $1 \mu \mathrm{m}(\mathbf{A}-\mathbf{F}) ; 20 \mu \mathrm{m}(\mathbf{G}-\mathbf{L})$. common mechanism for paresis and hypotonia in this group of conditions and a potential target for therapeutic intervention.

\section{Generalized Membranous and Cytoskeletal Defects Are Apparent in HACD1 CNM}

Sarcolemmal membrane abnormalities were also apparent in CNM-affected dogs, including dilated tubular and vesicular structures, membrane invaginations, and caveolae, which are similar to findings also apparent in other CNMs. ${ }^{18}$ Dysferlin and caveolin 3 associate with one another at the sarcolemma in mature skeletal muscle (Figure 5E): they localize to the developing T-tubule system and are involved in membrane trafficking and repair. ${ }^{60-62}$ Dysferlinopathies are associated with morphologic abnormalities in T-tubules. ${ }^{61}$ Several recent reports have documented abnormal localization of one or both of these proteins in autosomal CNMs ${ }^{14,21,63}$ : in particular, the cytoplasmic mislocalization and sarcolemmal invaginations apparent with dysferlin immunostaining in CNM-affected dogs in this study are similar to those reported in the autosomal recessive CNM associated with a BINI mutation in Great Dane dogs. ${ }^{21}$ In the current study, the pattern of cytoplasmic dysferlin staining often has a similar halo pattern to that observed in triad and oxidative stains, which might suggest that dysferlin localizes to the T-tubule system in these fibers for membrane trafficking and repair. Olby et $\mathrm{al}^{64}$ previously evaluated dysferlin in muscle from affected Labradors by Western blotting but did not detect any difference in total expression compared with controls.

Mislocalization of desmin, the major intermediate filament protein in skeletal muscle, is well documented in congenital myopathies in general and in CNMs in particular, where accumulations are associated with the centralized nucleus. ${ }^{1,2,65,66}$ A direct role in intermediate filament organization and maintenance, independent of defective phosphatase activity, has been documented for MTM1. ${ }^{67}$ Such cytoskeletal disorganization might also affect mitochondrial 

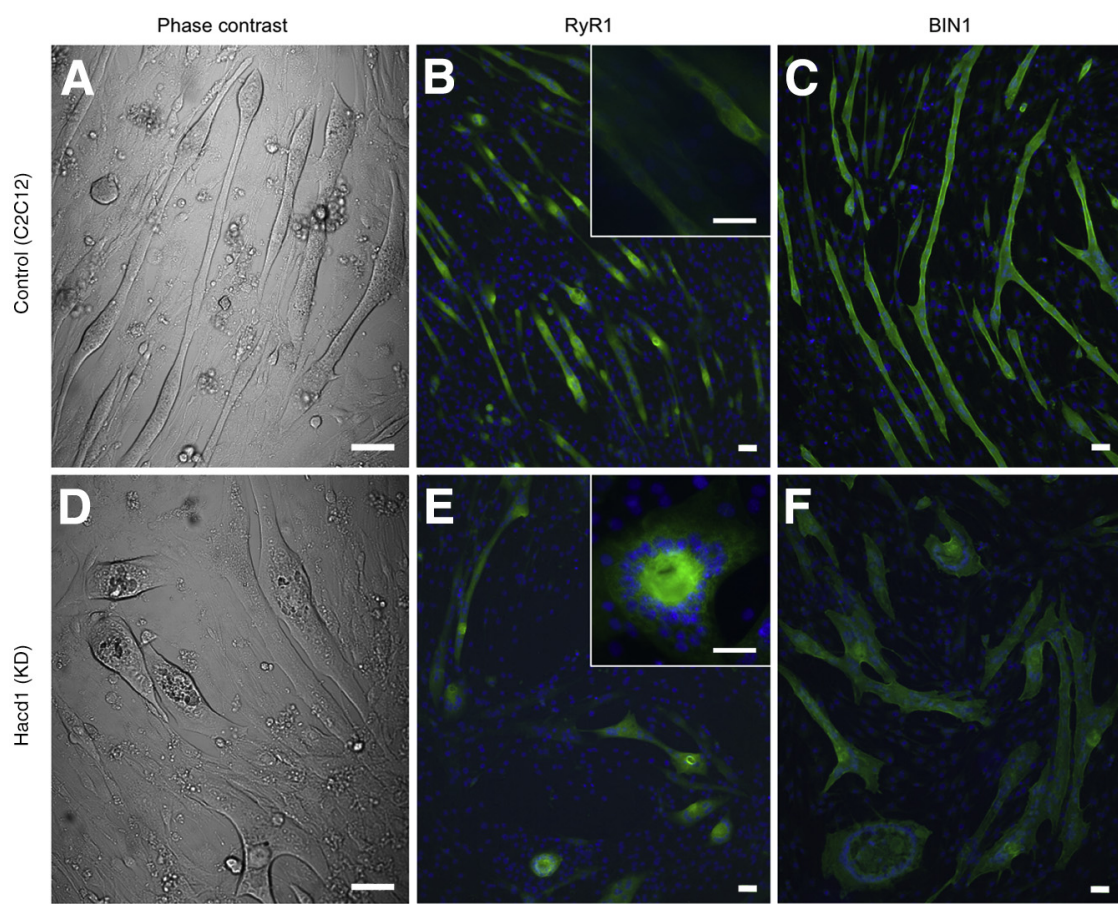

Figure 7 Morphology of control $(\mathbf{A}-\mathbf{C})$ and Hacd1 knockdown (KD) (D-F) myotubes at 12 days of differentiation. Phase contrast (A and $\mathbf{D})$ and wide-field immunofluorescence for ryanodine receptor (RyR1) ( $\mathbf{B}$ and $\mathbf{E}$, magnified in insets) and amphiphysin 2 (BIN1) ( $\mathbf{C}$ and $\mathbf{F}$ ) images showing the typical appearance of differentiated myotubes from both cell lines. D-F: Note the presence of intracellular vesicles (D) and rings of abnormally positioned nuclei ( $\mathbf{E}$ and $\mathbf{F}$ ) in the $\mathrm{KD}$ cell line. Scale bars $=50 \mu \mathrm{m}(\mathbf{A}-\mathbf{F}$, including insets $)$. localization and dynamics, ${ }^{67}$ perhaps explaining the abnormal mitochondrial distribution in HACDI CNM dogs. Progressive derangement of the contractile apparatus in $H A C D 1$ CNM dogs was indicated by the Z-line abnormalities, including accumulations and rods, and myofibrillar disorganization detected by electron microscopy and by the altered immunofluorescence staining pattern for sarcomeric myosin. Interestingly, in affected dogs, an area around the nucleus was often devoid of myofilaments regardless of positioning; this was not the case in the controls (Figure 6). BIN1 was recently found to bind both the nuclear envelope protein nesprin and the microtubule associated protein CLIP170, implicating a role for this CNM-associated protein in nuclear positioning. ${ }^{49}$ The interaction of the cytoskeleton and nuclear anchoring proteins, mechanisms that control myonuclear positioning, and the effect of abnormal nuclear internalization on muscle function are exciting areas for further research in CNM. ${ }^{68,69}$

Cytoarchitectural rearrangements have long been recognized as features of CNM and are often most obvious as unusual oxidative staining patterns (such as radial strands or necklaces). ${ }^{70}$ As in canine CNM, centralized nuclei are often surrounded by areas devoid of myofilaments that contain accumulations of mitochondria, glycogen, and SR membranes. ${ }^{70}$ Because intracellular systems do not exist in isolation, disorganization of internal membranes, in particular the SR, which interacts with numerous organelles, would be expected to affect positioning of other compartments. $^{20,55}$ Nonetheless, involvement of CNM-associated proteins in other complex protein-protein interactions is being increasingly documented. ${ }^{49,67,69,71}$ Extensive myofibrillar disorganization and nuclear internalization were initially described as part of the spectrum of congenital myopathies attributable to RYRl mutations, ${ }^{72}$ and more recently mutations in the $T T N$ gene encoding titin have been found to cause similar pathologic features. ${ }^{10} \mathrm{~A}$ mechanism for their development from areas of altered $\mathrm{Ca}^{2+}$ homeostasis has been proposed after following the progression of core development in Ryrl-mutant mice. ${ }^{53,73}$

\section{Disrupted Lipid Metabolism and Membrane Trafficking Present a Unifying Pathophysiologic Model for CNMs}

There is compelling experimental evidence of aberrant membrane trafficking from models of classic (MTM1-, BIN1-, DNM2-, and RyRl-associated) CNMs. ${ }^{5,12,15,18,20,74}$ We propose that HACD1 deficiency affects VLCFA biosynthesis, ${ }^{32,34,40}$ leading to altered T-tubule, SR, or sarcolemmal membrane properties and ultimately progressive dysfunction and degeneration of these muscle membranes. VLCFAs are components of complex lipids with important structural and signaling roles. ${ }^{75}$ VLCFAsubstituted phosphatidylinositol is thought to be important in stabilizing highly curved membranes because the VLFACs might associate with both sides of the lipid bilayer and fill areas left void where carbon chains are unopposed because of extreme curvature. ${ }^{76}$ Blondelle et al ${ }^{40}$ recently documented alterations in VLCFAs and LCFAs and unsaturated and saturated fatty acid ratios in HACD1-deficient models, which reduced plasma membrane fluidity and resulted in defective membrane fusion and impaired early myogenesis. A similar mechanism could account for the abnormalities and progressive degeneration in T-tubule membranes because fusion is required for T-tubule 
development (elongation), repair, and maintenance. ${ }^{15}$ Defects in Phs1 (the yeast homologue of HACDI) led to abnormalities in a number of signaling molecules, in particular, reduced concentrations of complex sphingolipids and phosphatidylinositol monophosphates, which notably include phosphatidylinositol 3-phosphate, the major substrate of myotubularin. ${ }^{34}$ Membrane defects are emerging as consistent features in $\mathrm{CNM}^{3,18,74}$; therefore, these findings lend additional credence to the inclusion of canine CNM in this classification and validate it as a potential large animal model for translational research.

Lin et $\mathrm{al}^{35}$ and Blondelle et $\mathrm{al}^{40}$ documented severely impaired differentiation in HACD1-deficient myoblasts, but, in contrast, we reveal progressive age-associated muscle degeneration in CNM-affected dogs. Similarly, other CNMassociated proteins (MTM1 and BIN1) have roles in both development ${ }^{12,46,77,78}$ and maintenance ${ }^{11,13,17,66,79,80}$ of the complex membrane systems in mature muscle. In HACD1deficient dogs, early differentiation of muscle might be partially rescued by expression from various paralogous genes, in particular, other HACD enzymes (such as ubiquitously expressed HACD2/PTPLB or HACD3/PTPLAD1) ${ }^{34}$ and perhaps maternal delivery of VLCFA via the placenta and milk. ${ }^{81}$ We hypothesize that culture conditions used in this study overcame a defect in cell fusion, allowing us to detect (at a later stage of differentiation) defective internal membrane system formation or maintenance.

Mutation of HACDI in humans has been reported in a single consanguineous family and resulted in a congenital myopathy with fiber-type disproportion. ${ }^{32}$ Patients had neonatal hypotonia and a severely myopathic phenotype that gradually improve: the single case evaluated in adulthood had normal gait; however, the absence of myotatic reflexes persisted. This finding is distinct to the progressive clinical course observed during growth in dogs with HACD1 deficiency, which is perhaps attributed to different effects of the two mutations on expression and function of HACD1: canine CNM is caused by an insertion that affects splicing and reduces expression of normal isoforms to $<1 \%$ of normal, ${ }^{29}$ whereas in the human family there was a Cterminal nonsense mutation that reduced expression to $30 \%$ of normal levels and produced an abnormally glycosylated protein. ${ }^{32}$ Alternatively, species-related phenotypic variation may relate to differences in dietary fatty acid composition and lipid metabolism or muscle development (the latter has been suggested for MTM1 mutations in humans, mice, and dogs to explain a similar discrepancy in age of onset and progression ${ }^{13}$ ). Pathologic features associated with the human HACD1 mutation, in common with canine CNM, included the presence of hypotrophic fibers and a marked a predominance of type 1 myofibers. ${ }^{32}$ An increased proportion of centralized nuclei was not identified, but pathologic description was limited to two patients with muscle biopsy samples taken at a young age (1 to 2 years). The proportion of centralized nuclei in affected dogs is often not markedly elevated at the time of diagnosis ${ }^{31}$ and increases progressively over time; the same might be true in affected human patients as they age. Dogs, mice, ${ }^{40}$ and humans $^{32}$ with loss-of-function mutations in HACD1 have congenital myopathy with several shared clinical and pathologic features: HACD1 should therefore be considered a candidate gene for congenital fiber-type disproportion syndromes and congenital and CNMs in humans and other species.

In conclusion, dogs with HACD1 deficiency have ultrastructural abnormalities in membranes, in particular in T-tubules and SR, a hallmark of classic CNM that is thought to be key factor in human disease symptoms and pathogenesis. In addition, they also replicate a number of other prominent pathologic features of this group of disorders. Our confirmation of this additional gene/protein as a cause of CNM enhances our understanding of the pathogenesis of these disorders. Furthermore, cementing the link between HACD1 deficiency and CNM provides mechanistic insight into the role of HACD/VLCFA in membrane trafficking and tubuloreticular membrane maintenance in muscle, which is of fundamental and pressing importance now that this gene has been linked to a congenital myopathy in humans. ${ }^{32}$ Finally, our work helps explain the profound and progressive paresis observed in the most prevalent inherited myopathy of Labrador retrievers, the most popular dog breed worldwide.

\section{Acknowledgments}

We thank Dr. Ruby Chang (Royal Veterinary College) for statistical support; Dr. Susan Brown and Claire Massey (Royal Veterinary College) for advice on histologic and histochemical staining and electron microscopy; Laurent Guillaud for technical assistance; and Dr. Pablo Aguilar, Xavier Cauchois, and the team who care for the dogs at the Neurobiology Laboratory at the Alfort School of Veterinary Medicine.

\section{Supplemental Data}

Supplemental material for this article can be found at http://dx.doi.org/10.1016/j.ajpath.2016.10.002.

\section{References}

1. Dubowitz V, Sewry C: Muscle Biopsy: A Practical Approach. ed 3. Philadelphia, PA, Elsevier, Saunders, 2007

2. Romero N, Laporte J. Centronucelar myopathies. Muscle Disease: Pathology and Genetics. Edited by Goebel HH, Sewry CA Weller RO. ed 2. Chichester: Wiley-Blackwell, 2013. pp. 134-144

3. Jungbluth H, Gautel M: Pathogenic mechanisms in centronuclear myopathies. Front Aging Neurosci 2014, 6:339

4. Laporte J, Hu LJ, Kretz C, Mandel JL, Kioschis P, Coy JF, Klauck SM, Poustka A, Dahl N: A gene mutated in X-linked myotubular myopathy defines a new putative tyrosine phosphatase family conserved in yeast. Nat Genet 1996, 13:175-182

5. Nicot AS, Toussaint A, Tosch V, Kretz C, Wallgren-Pettersson C, Iwarsson E, Kingston H, Garnier JM, Biancalana V, Oldfors A, 
Mandel JL, Laporte J: Mutations in amphiphysin 2 (BIN1) disrupt interaction with dynamin 2 and cause autosomal recessive centronuclear myopathy. Nat Genet 2007, 39:1134-1139

6. Bitoun M, Maugenre S, Jeannet PY, Lacene E, Ferrer X, Laforet P, Martin JJ, Laporte J, Lochmuller H, Beggs AH, Fardeau M, Eymard B, Romero NB, Guicheney P: Mutations in dynamin 2 cause dominant centronuclear myopathy. Nat Genet 2005, 37:1207-1209

7. Jungbluth H, Zhou H, Sewry CA, Robb S, Treves S, Bitoun M, Guicheney P, Buj-Bello A, Bonnemann C, Muntoni F: Centronuclear myopathy due to a de novo dominant mutation in the skeletal muscle ryanodine receptor (RYR1) gene. Neuromuscul Disord 2007, 17: 338-345

8. Majczenko K, Davidson AE, Camelo-Piragua S, Agrawal PB, Manfready RA, Li X, Joshi S, Xu J, Peng W, Beggs AH, Li JZ, Burmeister M, Dowling JJ: Dominant mutation of CCDC78 in a unique congenital myopathy with prominent internal nuclei and atypical cores. Am J Hum Genet 2012, 91:365-371

9. Agrawal PB, Pierson CR, Joshi M, Liu X, Ravenscroft G, Moghadaszadeh B, Talabere T, Viola M, Swanson LC, Haliloglu G, Talim B, Yau KS, Allcock RJ, Laing NG, Perrella MA, Beggs AH: SPEG interacts with myotubularin, and its deficiency causes centronuclear myopathy with dilated cardiomyopathy. Am J Hum Genet 2014, 95:218-226

10. Ceyhan-Birsoy O, Agrawal PB, Hidalgo C, Schmitz-Abe K, DeChene ET, Swanson LC, Soemedi R, Vasli N, Iannaccone ST, Shieh PB, Shur N, Dennison JM, Lawlor MW, Laporte J, Markianos K, Fairbrother WG, Granzier H, Beggs AH: Recessive truncating titin gene, TTN, mutations presenting as centronuclear myopathy. Neurology 2013, 81:1205-1214

11. Al-Qusairi L, Weiss N, Toussaint A, Berbey C, Messaddeq N, Kretz C, Sanoudou D, Beggs AH, Allard B, Mandel JL, Laporte J, Jacquemond V, Buj-Bello A: T-tubule disorganization and defective excitation-contraction coupling in muscle fibers lacking myotubularin lipid phosphatase. Proc Natl Acad Sci U S A 2009, 106: $18763-18768$

12. Dowling JJ, Vreede AP, Low SE, Gibbs EM, Kuwada JY, Bonnemann CG, Feldman EL: Loss of myotubularin function results in T-tubule disorganization in zebrafish and human myotubular myopathy. PLoS Genet 2009, 5:e1000372

13. Beggs AH, Bohm J, Snead E, Kozlowski M, Maurer M, Minor K, Childers MK, Taylor SM, Hitte C, Mickelson JR, Guo LT, Mizisin AP, Buj-Bello A, Tiret L, Laporte J, Shelton GD: MTM1 mutation associated with X-linked myotubular myopathy in Labrador Retrievers. Proc Natl Acad Sci U S A 2010, 107:14697-14702

14. Durieux AC, Vignaud A, Prudhon B, Viou MT, Beuvin M, Vassilopoulos S, Fraysse B, Ferry A, Laine J, Romero NB, Guicheney P, Bitoun M: A centronuclear myopathy-dynamin 2 mutation impairs skeletal muscle structure and function in mice. Hum Mol Genet 2010, 19:4820-4836

15. Al-Qusairi L, Laporte J: T-tubule biogenesis and triad formation in skeletal muscle and implication in human diseases. Skelet Muscle 2011, 1:26

16. Cowling BS, Toussaint A, Amoasii L, Koebel P, Ferry A, Davignon L, Nishino I, Mandel JL, Laporte J: Increased expression of wild-type or a centronuclear myopathy mutant of dynamin 2 in skeletal muscle of adult mice leads to structural defects and muscle weakness. Am J Pathol 2011, 178:2224-2235

17. Tjondrokoesoemo A, Park KH, Ferrante C, Komazaki S, Lesniak S, Brotto M, Ko JK, Zhou J, Weisleder N, Ma J: Disrupted membrane structure and intracellular $\mathrm{Ca}(2)(+)$ signaling in adult skeletal muscle with acute knockdown of Bin1. PLoS One 2011, 6:e25740

18. Toussaint A, Cowling BS, Hnia K, Mohr M, Oldfors A, Schwab Y, Yis U, Maisonobe T, Stojkovic T, Wallgren-Pettersson C, Laugel V, Echaniz-Laguna A, Mandel JL, Nishino I, Laporte J: Defects in amphiphysin 2 (BIN1) and triads in several forms of centronuclear myopathies. Acta Neuropathol 2011, 121:253-266
19. Hnia K, Kretz C, Amoasii L, Bohm J, Liu X, Messaddeq N, Qu CK, Laporte J: Primary T-tubule and autophagy defects in the phosphoinositide phosphatase Jumpy/MTMR14 knockout mice muscle. Adv Biol Regul 2012, 52:98-107

20. Amoasii L, Hnia K, Chicanne G, Brech A, Cowling BS, Muller MM, Schwab Y, Koebel P, Ferry A, Payrastre B, Laporte J: Myotubularin and PtdIns3P remodel the sarcoplasmic reticulum in muscle in vivo. J Cell Sci 2013, 126:1806-1819

21. Bohm J, Vasli N, Maurer M, Cowling B, Shelton GD, Kress W, Toussaint A, Prokic I, Schara U, Anderson TJ, Weis J, Tiret L, Laporte J: Altered splicing of the BIN1 muscle-specific exon in humans and dogs with highly progressive centronuclear myopathy. PLoS Genet 2013, 9:e1003430

22. Zhou H, Rokach O, Feng L, Munteanu I, Mamchaoui K, Wilmshurst JM, Sewry C, Manzur AY, Pillay K, Mouly V, Duchen M, Jungbluth H, Treves S, Muntoni F: RyR1 deficiency in congenital myopathies disrupts excitation-contraction coupling. Hum Mutat 2013, 34:986-996

23. Durieux AC, Vassilopoulos S, Laine J, Fraysse B, Brinas L, Prudhon B, Castells J, Freyssenet D, Bonne G, Guicheney P, Bitoun M: A centronuclear myopathy-dynamin 2 mutation impairs autophagy in mice. Traffic 2012, 13:869-879

24. Kramer JW, Hegreberg GA, Bryan GM, Meyers K, Ott RL: A muscle disorder of Labrador retrievers characterized by deficiency of type II muscle fibers. J Am Vet Med Assoc 1976, 169:817-820

25. Shelton GD: What's new in muscle and peripheral nerve diseases? Vet Comp Orthop Traumatol 2007, 20:249-255

26. Maurer M, Mary J, Guillaud L, Fender M, Pele M, Bilzer T, Olby N, Penderis J, Shelton GD, Panthier JJ, Thibaud JL, Barthelemy I, Aubin-Houzelstein G, Blot S, Hitte C, Tiret L: Centronuclear myopathy in Labrador retrievers: a recent founder mutation in the PTPLA gene has rapidly disseminated worldwide. PLoS One 2012, 7 : e46408

27. Blot S, Tiret L, Devillaire A, Fardeau M, Dreyfus PA: Phenotypic description of a canine centronuclear myopathy. J Neurol Sci 2002, 199:S9

28. Tiret L, Blot S, Kessler JL, Gaillot H, Breen M, Panthier JJ: The cnm locus, a canine homologue of human autosomal forms of centronuclear myopathy, maps to chromosome 2. Hum Genet 2003, 113 : 297-306

29. Pelé M, Tiret L, Kessler JL, Blot S, Panthier JJ: SINE exonic insertion in the PTPLA gene leads to multiple splicing defects and segregates with the autosomal recessive centronuclear myopathy in dogs. Hum Mol Genet 2005, 14:1417-1427

30. McKerrell R, Braund KG: Hereditary myopathy in labrador retrievers: clinical variations. J Small Anim Pract 1987, 28: 479-489

31. McKerrell RE, Braund KG: Hereditary myopathy in labrador retrievers: a morphologic study. Vet Pathol 1986, 23:411-417

32. Muhammad E, Reish O, Ohno Y, Scheetz T, Deluca A, Searby C, Regev M, Benyamini L, Fellig Y, Kihara A, Sheffield VC, Parvari R: Congenital myopathy is caused by mutation of HACD1. Hum Mol Genet 2013, 22:5229-5236

33. Uwanogho DA, Hardcastle Z, Balogh P, Mirza G, Thornburg KL, Ragoussis J, Sharpe PT: Molecular cloning, chromosomal mapping, and developmental expression of a novel protein tyrosine phosphatase-like gene. Genomics 1999, 62:406-416

34. Ikeda M, Kanao Y, Yamanaka M, Sakuraba H, Mizutani Y, Igarashi $\mathrm{Y}$, Kihara A: Characterization of four mammalian 3hydroxyacyl-CoA dehydratases involved in very long-chain fatty acid synthesis. FEBS Lett 2008, 582:2435-2440

35. Lin X, Yang X, Li Q, Ma Y, Cui S, He D, Schwartz RJ, Chang J: Protein tyrosine phosphatase-like A regulates myoblast proliferation and differentiation through $\mathrm{MyoG}$ and the cell cycling signaling pathway. Mol Cell Biol 2012, 32:297-308

36. Denic V, Weissman JS: A molecular caliper mechanism for determining very long-chain fatty acid length. Cell 2007, 130:663-677 
37. Kihara A, Sakuraba H, Ikeda M, Denpoh A, Igarashi Y: Membrane topology and essential amino acid residues of Phs1, a 3-hydroxyacylCoA dehydratase involved in very long-chain fatty acid elongation. $\mathrm{J}$ Biol Chem 2008, 283:11199-11209

38. Sabbadini RA, Danieli-Betto D, Betto R: The role of sphingolipids in the control of skeletal muscle function: a review. Ital J Neurol Sci 1999, 20:423-430

39. Balla T: Phosphoinositides: tiny lipids with giant impact on cell regulation. Physiol Rev 2013, 93:1019-1137

40. Blondelle J, Ohno Y, Gache V, Guyot S, Storck S, BlanchardGutton N, Barthelemy I, Walmsley G, Rahier A, Gadin S, Maurer M, Guillaud L, Prola A, Ferry A, Aubin-Houzelstein G, Demarquoy J, Relaix F, Piercy RJ, Blot S, Kihara A, Tiret L, Pilot-Storck F: HACD1, a regulator of membrane composition and fluidity, promotes myoblast fusion and skeletal muscle growth. J Mol Cell Biol 2015, 7: 429-440

41. Bach L, Michaelson LV, Haslam R, Bellec Y, Gissot L, Marion J, Da Costa M, Boutin JP, Miquel M, Tellier F, Domergue F, Markham JE, Beaudoin F, Napier JA, Faure JD: The very-long-chain hydroxy fatty acyl-CoA dehydratase PASTICCINO2 is essential and limiting for plant development. Proc Natl Acad Sci U S A 2008, 105: $14727-14731$

42. Molino D, Van der Giessen E, Gissot L, Hematy K, Marion J, Barthelemy J, Bellec Y, Vernhettes S, Satiat-Jeunemaitre B, Galli T, Tareste D, Faure JD: Inhibition of very long acyl chain sphingolipid synthesis modifies membrane dynamics during plant cytokinesis. Biochim Biophys Acta 2014, 1842:1422-1430

43. Walmsley GL, Arechavala-Gomeza V, Fernandez-Fuente M, Burke MM, Nagel N, Holder A, Stanley R, Chandler K, Marks SL, Muntoni F, Shelton GD, Piercy RJ: A duchenne muscular dystrophy gene hot spot mutation in dystrophin-deficient cavalier king charles spaniels is amenable to exon 51 skipping. PLoS One 2010, 5:e8647

44. Pfaffl MW, Horgan GW, Dempfle L: Relative expression software tool (REST) for group-wise comparison and statistical analysis of relative expression results in real-time PCR. Nucleic Acids Res 2002, 30:e36

45. Nishimura M, Nikawa T, Kawano Y, Nakayama M, Ikeda M: Effects of dimethyl sulfoxide and dexamethasone on mRNA expression of housekeeping genes in cultures of $\mathrm{C} 2 \mathrm{C} 12$ myotubes. Biochem Biophys Res Commun 2008, 367:603-608

46. Lee E, Marcucci M, Daniell L, Pypaert M, Weisz OA, Ochoa GC, Farsad K, Wenk MR, De Camilli P: Amphiphysin 2 (Bin1) and Ttubule biogenesis in muscle. Science 2002, 297:1193-1196

47. Royer B, Hnia K, Gavriilidis C, Tronchere H, Tosch V, Laporte J: The myotubularin-amphiphysin 2 complex in membrane tubulation and centronuclear myopathies. EMBO Rep 2013, 14:907-915

48. Picas L, Viaud J, Schauer K, Vanni S, Hnia K, Fraisier V, Roux A, Bassereau P, Gaits-Iacovoni F, Payrastre B, Laporte J, Manneville JB, Goud B: BIN1/M-Amphiphysin2 induces clustering of phosphoinositides to recruit its downstream partner dynamin. Nat Commun 2014, 5:5647

49. D’Alessandro M, Hnia K, Gache V, Koch C, Gavriilidis C, Rodriguez D, Nicot AS, Romero NB, Schwab Y, Gomes E, Labouesse M, Laporte J: Amphiphysin 2 orchestrates nucleus positioning and shape by linking the nuclear envelope to the actin and microtubule cytoskeleton. Dev Cell 2015, 35:186-198

50. Strbenc M, Smerdu V, Pogacnik A, Fazarinc G: Myosin heavy chain isoform transitions in canine skeletal muscles during postnatal growth. J Anat 2006, 209:149-163

51. Fugier C, Klein AF, Hammer C, Vassilopoulos S, Ivarsson Y, Toussaint A, Tosch V, Vignaud A, Ferry A, Messaddeq N, Kokunai Y, Tsuburaya R, de la Grange P, Dembele D, Francois V, Precigout G, Boulade-Ladame C, Hummel MC, Lopez de Munain A, Sergeant N, Laquerriere A, Thibault C, Deryckere F, Auboeuf D, Garcia L, Zimmermann P, Udd B, Schoser B, Takahashi MP, Nishino I, Bassez G, Laporte J, Furling D, Charlet-Berguerand N: Misregulated alternative splicing of BIN1 is associated with T tubule alterations and muscle weakness in myotonic dystrophy. Nat Med 2011, 17:720-725

52. Dowling JJ, Low SE, Busta AS, Feldman EL: Zebrafish MTMR14 is required for excitation-contraction coupling, developmental motor function and the regulation of autophagy. Hum Mol Genet 2010, 19: $2668-2681$

53. Boncompagni S, Rossi AE, Micaroni M, Hamilton SL, Dirksen RT, Franzini-Armstrong C, Protasi F: Characterization and temporal development of cores in a mouse model of malignant hyperthermia. Proc Natl Acad Sci U S A 2009, 106:21996-22001

54. Razzaq A, Robinson IM, McMahon HT, Skepper JN, Su Y, Zelhof AC, Jackson AP, Gay NJ, O'Kane CJ: Amphiphysin is necessary for organization of the excitation-contraction coupling machinery of muscles, but not for synaptic vesicle endocytosis in Drosophila. Genes Dev 2001, 15:2967-2979

55. Franzini-Armstrong C, Engel A: Skeletal muscle: architecture of membrane systems. Edited by Hill J, Olson E. In Muscle: Fundamental Biology and Mechanisms of Disease. London, UK: Elsevier Academic Press, 2012. pp. 763-774

56. Butler MH, David C, Ochoa GC, Freyberg Z, Daniell L, Grabs D, Cremona O, De Camilli P: Amphiphysin II (SH3P9; BIN1), a member of the amphiphysin/Rvs family, is concentrated in the cortical cytomatrix of axon initial segments and nodes of ranvier in brain and around T tubules in skeletal muscle. J Cell Biol 1997, 137: $1355-1367$

57. Parton RG, Way M, Zorzi N, Stang E: Caveolin-3 associates with developing T-tubules during muscle differentiation. J Cell Biol 1997, 136:137-154

58. Carozzi AJ, Ikonen E, Lindsay MR, Parton RG: Role of cholesterol in developing T-tubules: analogous mechanisms for T-tubule and caveolae biogenesis. Traffic 2000, 1:326-341

59. Nori A, Valle G, Bortoloso E, Turcato F, Volpe P: Calsequestrin targeting to sarcoplasmic reticulum of skeletal muscle fibers. Am J Physiol Cell Physiol 2006, 291:C245-C253

60. Bansal D, Miyake K, Vogel SS, Groh S, Chen CC, Williamson R, McNeil PL, Campbell KP: Defective membrane repair in dysferlindeficient muscular dystrophy. Nature 2003, 423:168-172

61. Klinge L, Harris J, Sewry C, Charlton R, Anderson L, Laval S, Chiu YH, Hornsey M, Straub V, Barresi R, Lochmuller H, Bushby K: Dysferlin associates with the developing T-tubule system in rodent and human skeletal muscle. Muscle Nerve 2010, 41:166-173

62. Sinha B, Koster D, Ruez R, Gonnord P, Bastiani M, Abankwa D, Stan RV, Butler-Browne G, Vedie B, Johannes L, Morone N, Parton RG, Raposo G, Sens P, Lamaze C, Nassoy P: Cells respond to mechanical stress by rapid disassembly of caveolae. Cell 2011, 144: 402-413

63. Bohm J, Biancalana V, Malfatti E, Dondaine N, Koch C, Vasli N, Kress W, Strittmatter M, Taratuto AL, Gonorazky H, Laforet P, Maisonobe T, Olive M, Gonzalez-Mera L, Fardeau M, Carriere N, Clavelou P, Eymard B, Bitoun M, Rendu J, Faure J, Weis J, Mandel JL, Romero NB, Laporte J: Adult-onset autosomal dominant centronuclear myopathy due to BIN1 mutations. Brain 2014, 137: $3160-3170$

64. Olby NJ, Sharp NJ, Anderson LV, Kunkel LM, Bonnemann CG: Evaluation of the dystrophin-glycoprotein complex, alpha-actinin, dysferlin and calpain 3 in an autosomal recessive muscular dystrophy in labrador retrievers. Neuromuscul Disord 2001, 11:41-49

65. Manta P, Mamali I, Zambelis T, Aquaviva T, Kararizou E, Kalfakis N: Immunocytochemical study of cytoskeletal proteins in centronuclear myopathies. Acta Histochem 2006, 108:271-276

66. Joubert R, Vignaud A, Le M, Moal C, Messaddeq N, Buj-Bello A: Site-specific Mtm1 mutagenesis by an AAV-Cre vector reveals that myotubularin is essential in adult muscle. Hum Mol Genet 2013, 22 : $1856-1866$

67. Hnia K, Tronchere H, Tomczak KK, Amoasii L, Schultz P, Beggs AH, Payrastre B, Mandel JL, Laporte J: Myotubularin controls desmin intermediate filament architecture and mitochondrial 
dynamics in human and mouse skeletal muscle. J Clin Invest 2011, 121:70-85

68. Metzger T, Gache V, Xu M, Cadot B, Folker ES, Richardson BE, Gomes ER, Baylies MK: MAP and kinesin-dependent nuclear positioning is required for skeletal muscle function. Nature 2012, 484: $120-124$

69. Falcone S, Roman W, Hnia K, Gache V, Didier N, Laine J, Aurade F, Marty I, Nishino I, Charlet-Berguerand N, Romero NB, Marazzi G, Sassoon D, Laporte J, Gomes ER: N-WASP is required for Amphiphysin-2/BIN1-dependent nuclear positioning and triad organization in skeletal muscle and is involved in the pathophysiology of centronuclear myopathy. EMBO Mol Med 2014, 6:1455-1475

70. Romero NB: Centronuclear myopathies: a widening concept. Neuromuscul Disord 2010, 20:223-228

71. Ferguson SM, De Camilli P: Dynamin, a membrane-remodelling GTPase. Nat Rev Mol Cell Biol 2012, 13:75-88

72. Bevilacqua JA, Monnier N, Bitoun M, Eymard B, Ferreiro A, Monges S, Lubieniecki F, Taratuto AL, Laquerriere A, Claeys KG, Marty I, Fardeau M, Guicheney P, Lunardi J, Romero NB: Recessive RYR1 mutations cause unusual congenital myopathy with prominent nuclear internalization and large areas of myofibrillar disorganization. Neuropathol Appl Neurobiol 2011, 37:271-284

73. Jungbluth H, Dowling JJ, Ferreiro A, Muntoni F: 182nd ENMC International Workshop: RYR1-related myopathies, 15-17th April 2011, Naarden, The Netherlands. Neuromuscul Disord 2012, 22:453-462

74. Cowling BS, Toussaint A, Muller J, Laporte J: Defective membrane remodeling in neuromuscular diseases: insights from animal models. PLoS Genet 2012, 8:e1002595
75. Kihara A: Very long-chain fatty acids: elongation, physiology and related disorders. J Biochem 2012, 152:387-395

76. Schneiter R, Brugger B, Amann CM, Prestwich GD, Epand RF, Zellnig G, Wieland FT, Epand RM: Identification and biophysical characterization of a very-long-chain-fatty-acid-substituted phosphatidylinositol in yeast subcellular membranes. Biochem J 2004, 381: 941-949

77. Lawlor MW, Alexander MS, Viola MG, Meng H, Joubert R, Gupta V, Motohashi N, Manfready RA, Hsu CP, Huang P, BujBello A, Kunkel LM, Beggs AH, Gussoni E: Myotubularin-deficient myoblasts display increased apoptosis, delayed proliferation, and poor cell engraftment. Am J Pathol 2012, 181:961-968

78. Shichiji M, Biancalana V, Fardeau M, Hogrel J, Osawa M, Laporte J, Romero N: Extensive morphological and immunohistochemical characterization in myotubular myopathy. Brain Behav 2013, 3: 476-486

79. Buj-Bello A, Laugel V, Messaddeq N, Zahreddine H, Laporte J, Pellissier JF, Mandel JL: The lipid phosphatase myotubularin is essential for skeletal muscle maintenance but not for myogenesis in mice. Proc Natl Acad Sci U S A 2002, 99:15060-15065

80. Hamanaka K, Inami I, Wada $\mathrm{T}$, Mitsuhshi S, Noguchi S, Hayashi YK, Nishino I: Muscle from a 20-week-old myotubular myopathy fetus is not myotubular. Neuromuscul Disord 2016, 26 : 234-235

81. Helland IB, Smith L, Saarem K, Saugstad OD, Drevon CA: Maternal supplementation with very-long-chain n-3 fatty acids during pregnancy and lactation augments children's IQ at 4 years of age. Pediatrics 2003, 111:e39-e44 